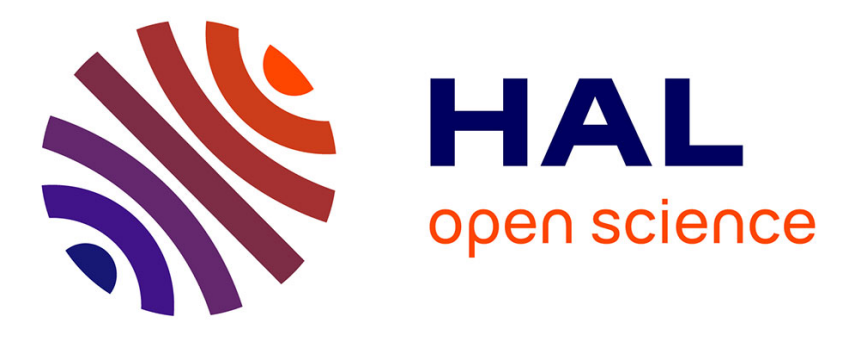

\title{
Vertical spectra of stratified turbulence at large horizontal scales
}

\author{
Andrea Maffioli
}

\section{To cite this version:}

Andrea Maffioli. Vertical spectra of stratified turbulence at large horizontal scales. Physical Review Fluids, 2017, 2, pp.104802. 10.1103/physrevfluids.2.104802 . hal-03157666

\section{HAL Id: hal-03157666 https://hal.science/hal-03157666}

Submitted on 10 Jun 2021

HAL is a multi-disciplinary open access archive for the deposit and dissemination of scientific research documents, whether they are published or not. The documents may come from teaching and research institutions in France or abroad, or from public or private research centers.
L'archive ouverte pluridisciplinaire HAL, est destinée au dépôt et à la diffusion de documents scientifiques de niveau recherche, publiés ou non, émanant des établissements d'enseignement et de recherche français ou étrangers, des laboratoires publics ou privés. 


\title{
Vertical spectra of stratified turbulence at large horizontal scales
}

\author{
Andrea Maffioli* \\ Linné Flow Centre, KTH Mechanics, Osquars Backe 18, 10044 Stockholm, Sweden
}

(Received 31 March 2017; published 19 October 2017)

\begin{abstract}
Stably stratified turbulence is investigated with the aim of increasing our limited understanding of the vertical structure of this type of turbulent flow. For strongly stratified turbulence there is a theoretical prediction that the energy spectra in the vertical direction of gravity are very steep, possessing the well-known form $E_{h}\left(k_{v}\right) \propto N^{2} k_{v}^{-3}$, where $N$ is the Brunt-Väisälä frequency and $k_{v}$ is the vertical wave number, but supporting evidence from experiments and numerical simulations is lacking. We conduct direct numerical simulation (DNS) with uniform background stratification and forcing at large scales. In order to consider the large anisotropic scales only, the vertical energy spectra are decomposed into large-scale vertical spectra $E_{\text {large }}\left(k_{v}\right)$ and small-scale vertical spectra $E_{\text {small }}\left(k_{v}\right)$ using a horizontal demarcation scale. We find that this approach gives results that are in close agreement with $E_{\text {large }}\left(k_{v}\right) \propto N^{2} k_{v}^{-3}$ for the DNS runs performed. This result holds approximately over the wave-number range $k_{b} \leqslant k_{v} \leqslant k_{\mathrm{oz}}$, where $k_{b}$ is the buoyancy wave number and $k_{\mathrm{oz}}$ is the Ozmidov wave number, in agreement with theory. Similarly, large-scale vertical spectra of potential energy are found to be $E_{p \text {, large }}\left(k_{v}\right) \propto N^{2} k_{v}^{-3}$, over a narrower range of wave numbers. The evidence supports the existence of a scale-by-scale balance between inertia and buoyancy occurring in strongly stratified turbulence at large horizontal scales. Finally, the current results are put in the context of ocean turbulence by making a comparison with measurements of vertical shear spectra made in the ocean interior.
\end{abstract}

DOI: 10.1103/PhysRevFluids.2.104802

\section{INTRODUCTION}

Natural flows in the atmosphere and in the oceans can be affected by a stable density stratification. The density stratification that is dynamically relevant is caused by vertical temperature gradients in the atmosphere and vertical temperature and salinity gradients in the oceans. The physical parameter that determines the relative strength of the stratification is the Froude number. In this paper we consider mainly turbulent flows and so we use a turbulence Froude number Fr $=\epsilon / N u_{h}^{2}$, where $\epsilon$ is the kinetic energy dissipation rate, $u_{h}$ is a horizontal turbulent velocity scale, and $N=\sqrt{-\frac{g}{\rho_{0}} \frac{d \bar{\rho}}{d z}}$ is the Brunt-Väisälä frequency, related to the background density gradient $\frac{d \bar{\rho}}{d z}$. The use of the turbulence Froude number was first proposed in the context of decaying stratified turbulence [1] and it is equivalent to a horizontal Froude number $\mathrm{Fr}_{h}=u_{h} / N \ell_{h}$, where $\ell_{h}$ is the horizontal length scale of the turbulence [2]. When the Froude number is low, Fr $\ll 1$, the dynamics is strongly affected by the buoyancy force and the flow develops in a layered structure that is a distinctive feature of stratified turbulence [3]. At high Reynolds number the flow can still be turbulent and three dimensional despite the strong vertical stability imposed by the stratification, and this flow regime, known as the strongly stratified turbulence regime, is the subject of the present study. The conditions to be in the strongly stratified turbulence regime are that $\mathrm{Fr} \ll 1$ and $\operatorname{Re}_{b} \gg 1$ concurrently, where $\operatorname{Re}_{b}=\epsilon / \nu N^{2}$ is the buoyancy Reynolds number, which needs to be large for viscous effects to be neglected [2]. In this regime, the turbulent layers have a vertical length scale $\ell_{v}$ that is of the order of the buoyancy length scale $\ell_{b}=u_{h} / N$ [4], a prediction which has been confirmed by several experimental and numerical investigations of the problem [2,5-8]. The physical meaning of this

*maffioli@mech.kth.se 
result is that inertia and buoyancy forces are in approximate balance since the vertical Froude number is $\mathrm{Fr}_{v}=u_{h} / N \ell_{v} \sim 1$ [8]. In the study by Billant and Chomaz [4] it was proposed that in the case of strongly stratified turbulence the above result holds on a scale-by-scale basis so that there should be continuously smaller layered structures of vertical scale $s_{v} \sim v_{h} / N$, where $s_{v}$ and $v_{h}$ are the local vertical length scale and horizontal velocity scale. This hypothesis implies that the local vertical Froude number $\operatorname{Fr}_{s_{v}}=v_{h} / N s_{v}$ should also be of order unity and that there is a balance between inertia and buoyancy forces over a range of scales, which we hence refer to as the "buoyancy-inertial range." In terms of vertical scales, this range should be $\ell_{b} \geqslant s_{v} \geqslant \ell_{\mathrm{oz}}$, where $\ell_{\mathrm{oz}}=\sqrt{\epsilon / N^{3}}$ is the Ozmidov length scale. At $s_{v}=\ell_{\mathrm{oz}}$ the local turbulence Froude number $\mathrm{Fr}_{\text {loc }}=\epsilon / N v_{h}^{2}$ is of order unity and there is a transition to isotropy, achieved at scales of motion much smaller than the Ozmidov length scale which are insensitive to the stratification $[9,10]$. Translated into Fourier space, the hypothesis of a buoyancy-inertial range allowed the derivation of the vertical spectrum of horizontal kinetic energy $E_{h}\left(k_{v}\right)[4]$ :

$$
E_{h}\left(k_{v}\right)=\alpha N^{2} k_{v}^{-3}
$$

where $\alpha$ is a constant of order unity. This form is valid over the range $k_{b} \leqslant k_{v} \leqslant k_{\mathrm{oz}}$, where the buoyancy wave number and Ozmidov wave number are simply defined as $k_{b}=1 / \ell_{b}=N / u_{h}$ and $k_{\mathrm{oz}}=1 / \ell_{\mathrm{oz}}=\sqrt{N^{3} / \epsilon}$. With the assumption of equipartition between kinetic and potential energy a similar expression was obtained for the vertical spectrum of potential energy in the buoyancy-inertial range, $E_{p}\left(k_{v}\right)=\beta N^{2} k_{v}^{-3}$, where $\beta$ is another constant of order unity [4]. Hence, the vertical energy spectra are very steep and are affected by the stratification $N$. On the other hand, the horizontal spectra of kinetic energy and of potential energy have been proposed to be $E_{h}\left(k_{h}\right) \sim \epsilon^{2 / 3} k_{h}^{-5 / 3}$ and $E_{p}\left(k_{h}\right) \sim\left(\epsilon_{p} / \epsilon^{1 / 3}\right) k_{h}^{-5 / 3}$ for $k_{\ell_{h}} \leqslant k_{h} \leqslant k_{\mathrm{oz}}$, independent of $N$ and where $k_{h}$ is the horizontal wave number, $\epsilon_{p}$ is the potential energy dissipation rate, and $k_{\ell_{h}}=1 / \ell_{h}$ corresponds to the largest horizontal scales [10]. While the evidence from recent numerical simulations is, as we will see, not in agreement with the vertical spectrum of Eq. (1), there is support for these forms of the horizontal spectra $[2,8,11,12]$. It is important to note that the wave-number ranges $k_{\ell_{h}} \leqslant k_{h} \leqslant k_{\mathrm{oz}}$ and $k_{b} \leqslant k_{v} \leqslant k_{\mathrm{oz}}$ correspond to the same dynamics within stratified turbulence, namely, to anisotropic layers with wave numbers going from $\left[k_{h}, k_{v}\right] \sim\left[k_{\ell_{h}}, k_{b}\right]$ to $\left[k_{h}, k_{v}\right] \sim\left[k_{\mathrm{oz}}, k_{\mathrm{oz}}\right]$ and which have a gradual reduction of the anisotropy.

The vertical spectrum in Eq. (1) is sometimes known as the "saturation spectrum" [13]. This is because it was previously derived in the context of atmospheric flows by assuming that turbulence is created by internal gravity waves going unstable due to shear or gravitational instability and eventually reaching a saturation condition close to the critical point for the onset of instability $[14,15]$. Vertical energy spectra measured in the free atmosphere (see Ref. [15] and references therein) and in the ocean [16,17] are consistent with the form given in Eq. (1). As shown in Fig. 1, measurements of the vertical shear spectrum $S\left(k_{v}\right)$ in the ocean gave a convincing result that $S\left(k_{v}\right) \approx N^{2} k_{v}^{-1}$ over a significant range of vertical wave numbers up to the Ozmidov wave number [17]. This form of the shear spectrum corresponds to a vertical energy spectrum $E_{h}\left(k_{v}\right) \approx 0.5 N^{2} k_{v}^{-3}$, consistent with Eq. (1) with $\alpha \approx 0.5$.

The positive match between the simple theoretical result and vertical energy spectra measured in the atmosphere and oceans supports the claim that strongly stratified turbulence is a relevant model for turbulence in the atmosphere and oceans in their interior regions [18]. However, many experiments and DNS have been conducted of stratified turbulence (see, e.g., Ref. [19] for experimental investigations and Refs. $[8,13,20]$ for numerical simulations) and these investigations have consistently been unable to reproduce vertical energy spectra in agreement with Eq. (1). Going more into the detail of recent studies with high-resolution simulations, the picture remains unclear since DNS of decaying stratified turbulence $[8,11]$ has given vertical spectra in compensated form, $E_{h}\left(k_{v}\right) /\left(N^{2} k_{v}^{-3}\right)$, which do not present a plateau in agreement with Eq. (1) but rather a broad maximum at high vertical wave numbers (the vertical spectra of the simulations in Ref. [11] were communicated to the author by P. Bartello [21]). In numerical simulations of forced stratified 


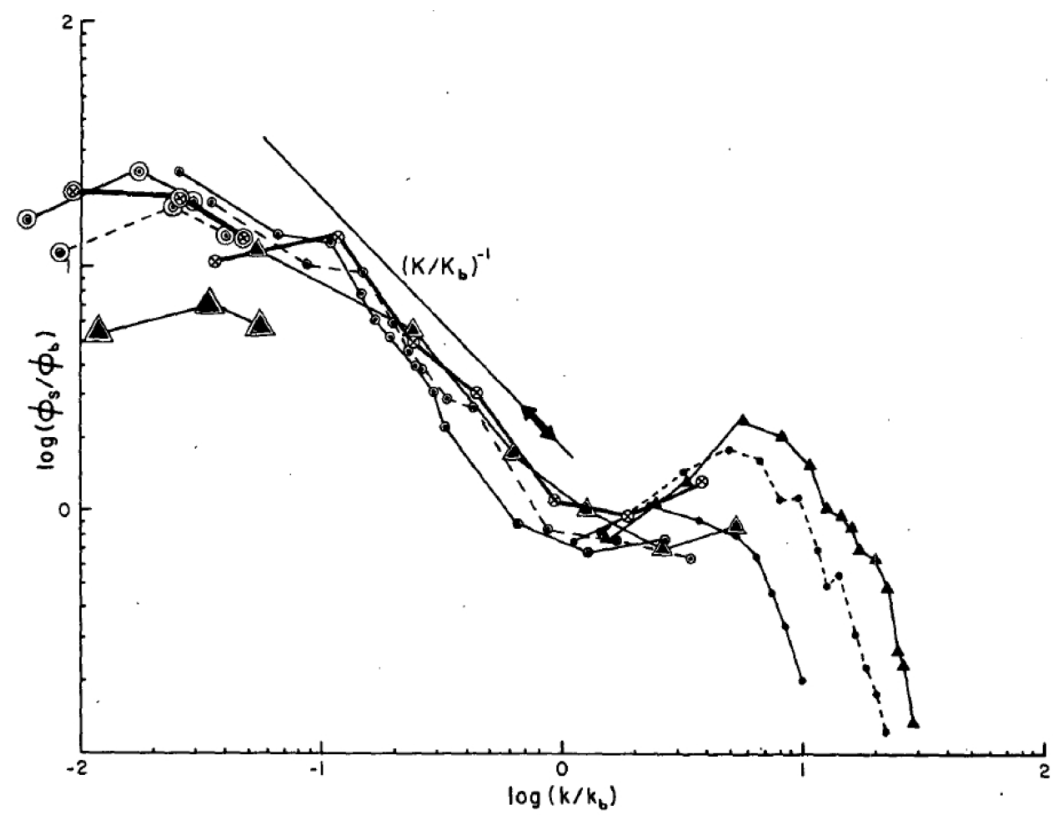

FIG. 1. Vertical spectra of vertical shear measured in the ocean by Gargett et al. [17]. The shear spectra are nondimensionalized by $\phi_{b}=\sqrt{\epsilon N}$ and the vertical wave number is nondimensionalized by the Ozmidov wave number (this is written as $k_{b}$ in their notation). The logarithm is in base 10 on both axes. Figure reproduced from Ref. [17] with permission, (c) American Meteorological Society.

turbulence with hyperviscosity [12] the vertical spectrum was close to $E_{h}\left(k_{v}\right) \propto k_{v}^{-3}$ in a single run while in the remaining runs the compensated vertical spectra presented a similar "bump" at high wave numbers. An analogous discrepancy was observed in the results from high-resolution DNS of forced stratified turbulence [22]. It is possible that the lack of clear evidence supporting Eq. (1) is due to the fact that the buoyancy-inertial range, if it exists, is very narrow since its width scales as $k_{\mathrm{oz}} / k_{b}=\left(N u_{h}^{2} / \epsilon\right)^{1 / 2}=\mathrm{Fr}^{-1 / 2}$. To date, experiments and numerical simulations of stratified turbulence at high Reynolds number have been limited to values of the Froude number in the range Fr $\approx 10^{-2}-10^{-1}[7,8,11]$, resulting in less than a decade of buoyancy-inertial range. In addition, it is expected that for high vertical wave numbers $k_{v}>k_{\mathrm{oz}}$ at which buoyancy effects are negligible, there is a range of scales over which we have homogeneous isotropic turbulence, with an associated vertical spectrum $E_{h}\left(k_{v}\right) \propto \epsilon^{2 / 3} k_{v}^{-5 / 3}$ up to the dissipation scales [2,15]. In two recent numerical studies of the transition to turbulence of a dipole vortex [23] and of strongly stratified turbulence [12] it was found that a composite vertical spectrum obtained by summing a term proportional to $N^{2} k_{v}^{-3}$ and a term proportional to $\epsilon^{2 / 3} k_{v}^{-5 / 3}$ gives a good comparison with results from the simulations. Such a composite spectrum describes the dynamics in the presence of an overlap region between the buoyancy-inertial range and the classical inertial range at small scales. However, an alternative explanation exists for the lack of evidence in favor of Eq. (1): vertical energy spectra shallower than $k_{v}^{-3}$ typically observed in simulations $[8,11,20]$ could entail that there is a gap in the theory of strongly stratified turbulence and that the vertical spectrum of Eq. (1) is incorrect. If this is the case, the vertical spectra observed in the atmosphere and oceans must be the consequence of a different set of dynamics, possibly more complicated than the simple model of strongly stratified turbulence with constant background stratification, as suggested in Ref. [13].

Shedding some light on this issue is the main motivation of the present investigation and we attempt to do this using results from high-resolution DNS of strongly stratified turbulence. The 
question driving this study is this: Can we recover vertical energy spectra showing the buoyancyinertial range in line with Eq. (1)?

To answer this question we explore the possibility of decomposing a field of strongly stratified turbulence into large scales and small scales. In stratified turbulence the large scales of motion are highly anisotropic while the small scales are approximately isotropic, and the decomposition is intended to isolate the two different sets of dynamics. In this paper the classical scale decomposition of isotropic turbulence [24] is adapted and used to obtain vertical energy spectra conditioned over large horizontal scales, which are strongly affected by buoyancy, and the results are then compared to the vertical spectrum in Eq. (1). Vertical energy spectra at large horizontal scales have been computed previously in simulations of stratified turbulence using a filter scale close to the buoyancy length scale $\ell_{b}$ but spectra significantly steeper than $k_{v}^{-3}$ were obtained [12,23].

This paper is organized as follows. In Sec. II the theory and analytical tools necessary to form large-scale vertical spectra are presented. Then in Sec. III the numerical methods of the DNS are given, including details on the simulations that were performed, and the results from the DNS concerning vertical spectra are presented in Sec. IV. Further implications of our results are discussed in Sec. V and the conclusions of the present study are summarized in Sec. VI.

\section{BACKGROUND AND THEORY}

\section{A. Scale decomposition of turbulent flows}

In the classical analysis of turbulence by Obukhov [24] a decomposition of the turbulent flow field into large scales and small scales based on a demarcation scale $\ell$ is introduced (see Ref. [25] for a complete account of the theory). In the analysis a field of homogeneous isotropic turbulence with velocity $\mathbf{u}$ is subdivided into a large-scale velocity field $\mathbf{u}_{\text {large }}$ and a small-scale velocity field $\mathbf{u}_{\text {small }}$. The scale decomposition is based on a filtering operation carried out in Fourier space using a single wave number $\mathcal{K}$. In essence, it consists of a sharp cutoff filter at the filter wave number $\mathcal{K}$, which is applied on $\hat{\mathbf{u}}(\mathbf{k})$, the Fourier transform of $\mathbf{u}(\mathbf{x})$. This means that the filter location is a sphere of radius $|\mathbf{k}|=\mathcal{K}$ in Fourier space, where $\mathbf{k}=\left[k_{x} k_{y} k_{z}\right]$ is the wave vector, and the associated filter scale is $\ell=1 / \mathcal{K}$. It is evident that in order for this decomposition to have a clear physical interpretation the turbulent flow field has to be isotropic. Hence, by considering only the contributions of wave numbers smaller than the filter wave number $\mathcal{K}$ we obtain the large-scale velocity field, whereas by considering only the contributions of wave numbers greater than $\mathcal{K}$ we obtain the small-scale velocity field, as follows:

$$
\begin{aligned}
& \mathbf{u}_{\text {large }}(\mathbf{x})=\sum_{|\mathbf{k}| \leqslant \mathcal{K}} \hat{\mathbf{u}}(\mathbf{k}) e^{i \mathbf{k} \cdot \mathbf{x}}, \\
& \mathbf{u}_{\text {small }}(\mathbf{x})=\sum_{|\mathbf{k}|>\mathcal{K}} \hat{\mathbf{u}}(\mathbf{k}) e^{i \mathbf{k} \cdot \mathbf{x}},
\end{aligned}
$$

and the velocity field can now be written as $\mathbf{u}(\mathbf{x})=\mathbf{u}_{\text {large }}(\mathbf{x})+\mathbf{u}_{\text {small }}(\mathbf{x})$.

\section{B. Scale decomposition of stratified turbulence}

We attempt at providing a similar formal decomposition into large scales and small scales for the case of stratified turbulence. The anisotropy of this turbulent flow means that we cannot define a single filter wave number $\mathcal{K}$ corresponding to a single filter scale $\ell$. While the flow is anisotropic and develops in layers, it is statistically axisymmetric about the vertical $z$ axis along which gravity acts and so there is no preferential horizontal direction. As a result, we should consider two wave numbers for our scale decomposition: a horizontal wave number $\mathcal{K}_{h}$ and a vertical wave number $\mathcal{K}_{v}$. More critically, the shape of the volume in Fourier space over which to sum now makes a difference to the final result and there is no obviously preferable choice. For example, one could choose a spheroid or a cylinder as the volume for the summation. If we choose a cylindrical volume, then we have the following expressions for the large-scale velocity field and the small-scale 
velocity field:

$$
\begin{gathered}
\mathbf{u}_{\text {large }}(\mathbf{x})=\sum_{k_{v} \leqslant \mathcal{K}_{v}} \sum_{k_{h} \leqslant \mathcal{K}_{h}} \hat{\mathbf{u}}(\mathbf{k}) e^{i \mathbf{k} \cdot \mathbf{x}}, \\
\mathbf{u}_{\text {small }}(\mathbf{x})=\sum_{k_{z}} \sum_{k_{h}>\mathcal{K}_{h}} \hat{\mathbf{u}}(\mathbf{k}) e^{i \mathbf{k} \cdot \mathbf{x}}+\sum_{k_{v}>\mathcal{K}_{v}} \sum_{k_{h} \leqslant \mathcal{K}_{h}} \hat{\mathbf{u}}(\mathbf{k}) e^{i \mathbf{k} \cdot \mathbf{x}},
\end{gathered}
$$

where we have introduced the vertical wave number $k_{v}=\left|k_{z}\right|$ and the horizontal wave number $k_{h}=\sqrt{k_{x}^{2}+k_{y}^{2}}$. We make this choice of the summation volume for simplicity and because it means that the horizontal and vertical directions are independent of one another and that we can define two constant filter wave numbers $\mathcal{K}_{h}$ and $\mathcal{K}_{v}$. On the other hand, if we had opted for a spheroid, the cutoff filter would be active on a surface described by $f\left(k_{h}, k_{v}\right)=0$ for which it would not be possible to define a single horizontal scale and a single vertical scale of the filtering, unambiguously. The scale-dependent decomposition described by Eqs. (4) and (5) also allows us to extend the analysis to consider the statistics of the large-scale velocity field in one of the two independent directions.

\section{The large-scale vertical spectrum}

In stratified turbulence it is necessary to consider horizontal and vertical spectra separately because of the anisotropy of the flow $[10,15]$. In this paper we focus on the vertical spectrum $E_{h}\left(k_{v}\right)$, which is a one-dimensional spectrum computed using only the two horizontal components of velocity $u$ and $v$ since the vertical velocity $w$ is on average much smaller than $u$ and $v$. Hence, the vertical spectrum is defined as

$$
E_{h}\left(k_{v}\right)=\sum_{\substack{k_{x}, k_{y} \\\left|k_{z}\right|=k_{v}}} \frac{1}{2}\left(\hat{u}(\mathbf{k}) \hat{u}^{*}(\mathbf{k})+\hat{v}(\mathbf{k}) \hat{v}^{*}(\mathbf{k})\right),
$$

where, for a given vertical wave number $k_{v}$, the summation is over two horizontal planes at $k_{z}= \pm k_{v}$. Similarly to other one-dimensional spectra, the vertical spectrum $E_{h}\left(k_{v}\right)$ at a given vertical wave number suffers from aliasing of Fourier modes at higher wave numbers, as discussed by Pope [26]. Considering Eq. (6), the contributions to $E_{h}\left(k_{v}\right)$ for a certain vertical wave number $k_{v}=k_{0}$ come from all horizontal wave numbers $k_{x}$ and $k_{y}$, including $\left|k_{x}\right|>k_{0}$ and $\left|k_{y}\right|>k_{0}$ so that these small scales are aliased to a vertical wave number $k_{0}$ corresponding to a larger scale. This can have an important effect in stratified turbulence, where the small scales of motion with large $k_{h}$ or alternatively large $k_{v}$ have a transition to isotropy (note that at high wave numbers approximate isotropy results in spherical symmetry in Fourier space). As illustrated in Fig. 2, even if we consider a vertical wave number $k_{v}<k_{\mathrm{oz}}$ the vertical spectrum $E_{h}\left(k_{v}\right)$ will have contributions that, beyond a critical horizontal wave number $k_{h}$, come from Fourier modes corresponding to small isotropic scales. This highlights the problem whereby using $E_{h}\left(k_{v}\right)$ we cannot obtain a definite measurement of the energy of the anisotropic scales in stratified turbulence (i.e., of the layers) as we only provide the value of $k_{v}$.

This limitation of $E_{h}\left(k_{v}\right)$ can be overcome if one considers vertical spectra after applying the scale decomposition. First of all, it should be noticed that the large-scale velocity field $\mathbf{u}_{\text {large }}$ given by Eq. (4) can be used to calculate two-point correlations but that clearly these will have missing information at small scales. This will also be true for the vertical spectrum $E_{h}\left(k_{v}\right)$ formed from this velocity field. If one, however, purposely chooses $\mathcal{K}_{v}=\infty$ and applies the low-pass filter operation of Eq. (4), it will be possible to calculate statistics of the resulting large-scale velocity field in the vertical direction, over all vertical scales of motion. We use a different notation for the large-scale velocity field obtained in this way,

$$
\mathbf{u}_{\text {large } \mathcal{H}}(\mathbf{x})=\sum_{k_{z}} \sum_{k_{h} \leqslant \mathcal{K}_{h}} \hat{\mathbf{u}}(\mathbf{k}) e^{i \mathbf{k} \cdot \mathbf{x}},
$$




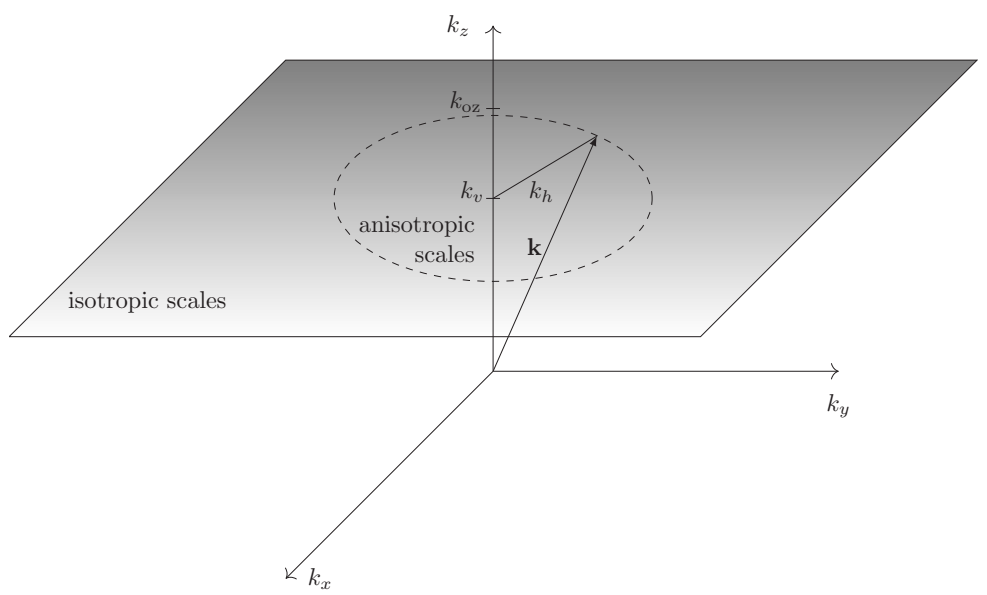

FIG. 2. Aliasing problem in the vertical spectrum in stratified turbulence. For a given vertical wave number $k_{v}, E_{h}\left(k_{v}\right)$ is formed by summing over all Fourier modes in the horizontal plane at $k_{z}=k_{v}$ (and at $k_{z}=-k_{v}$ ).

which is the velocity field at large horizontal scales. From $\mathbf{u}_{\text {large }} \mathcal{H}(\mathbf{x})$ we can calculate the onedimensional energy spectrum in the vertical direction:

$$
E_{\text {large }}\left(k_{v}\right)=\sum_{\substack{k_{x}, k_{y} \\\left|k_{z}\right|=k_{v}}} \frac{1}{2}\left(\hat{u}_{\text {large } \mathcal{H}}(\mathbf{k}) \hat{u}_{\text {large } \mathcal{H}}^{*}(\mathbf{k})+\hat{v}_{\text {large } \mathcal{H}}(\mathbf{k}) \hat{v}_{\text {large } \mathcal{H}}^{*}(\mathbf{k})\right)=\sum_{\substack{k_{h} \leqslant \mathcal{K}_{h} \\\left|k_{z}\right|=k_{v}}} \frac{1}{2}\left(\hat{u} \hat{u}^{*}+\hat{v} \hat{v}^{*}\right) .
$$

This vertical spectrum is a measure of the kinetic energy of turbulent structures with horizontal scales greater than the filter scale $L_{h}=1 / \mathcal{K}_{h}$ as a function of their vertical wave number. Hence, $E_{\text {large }}\left(k_{v}\right)$ is the vertical energy spectrum at large horizontal scales and we refer to it using the shorthand form large-scale vertical spectrum. Within this framework, we can similarly find an expression for a small-scale vertical spectrum of a field of stratified turbulence, obtained using a high-pass filter operation:

$$
E_{\text {small }}\left(k_{v}\right)=\sum_{\substack{k_{h}>\mathcal{K}_{h} \\\left|k_{z}\right|=k_{v}}} \frac{1}{2}\left(\hat{u} \hat{u}^{*}+\hat{v} \hat{v}^{*}\right)
$$

The full vertical spectrum can be obtained by summing the two spectra obtained from this decomposition, $E_{h}\left(k_{v}\right)=E_{\text {large }}\left(k_{v}\right)+E_{\text {small }}\left(k_{v}\right)$.

A similar decomposition can be applied to the buoyancy field, defined as $b=-\rho^{\prime} g / \rho_{0}$, where $\rho^{\prime}$ is the density perturbation away from the background density profile $\bar{\rho}(z)$ and $\rho_{0}$ is a reference density. From the buoyancy, the potential energy in stratified turbulence is obtained as $E_{P}=\left\langle b^{2}\right\rangle /\left(2 N^{2}\right)$, where $\langle\cdots\rangle$ denotes a volume average over the physical domain. The vertical spectrum of potential energy $E_{p}\left(k_{v}\right)$ is then defined analogously to the vertical energy spectrum given in Eq. (6). Applying the scale decomposition only in the horizontal direction, we can obtain expressions for the large-scale and small-scale vertical spectra of potential energy,

$$
\begin{aligned}
& E_{p, \text { large }}\left(k_{v}\right)=\sum_{\substack{k_{h} \leqslant \mathcal{K}_{h} \\
\left|k_{z}\right|=k_{v}}} \frac{1}{2 N^{2}} \hat{b} \hat{b}^{*}, \\
& E_{p, \text { small }}\left(k_{v}\right)=\sum_{\substack{k_{h}>\mathcal{K}_{h} \\
\left|k_{z}\right|=k_{v}}} \frac{1}{2 N^{2}} \hat{b} \hat{b}^{*},
\end{aligned}
$$


and the full vertical spectrum of potential energy can be recovered as $E_{p}\left(k_{v}\right)=E_{p \text {, large }}\left(k_{v}\right)+$ $E_{p \text {, small }}\left(k_{v}\right)$.

\section{Selection of the filter scale}

It is the case with most filtering operations in physics problems that the results of the filtering are highly dependent on the specific filter scale that is selected, in addition to the particular shape of the filter. The case of filtering a field of stratified turbulence to obtain the large-scale velocity field defined in Eq. (7) is no exception to this rule. Consequently, a reliable filter horizontal wave number $\mathcal{K}_{h}$ and corresponding filter scale $L_{h}$ should be chosen if we want the resulting large-scale vertical spectrum $E_{\text {large }}\left(k_{v}\right)$ to be accurately describing the buoyancy-inertial range of strongly stratified turbulence. Based on the theoretical prediction that the Ozmidov length scale is the relevant demarcation scale between large scales and small scales we could choose $\mathcal{K}_{h}=k_{\mathrm{oz}}$. However, the definition of the Ozmidov length scale [9] is based on order of magnitude arguments so we look for a different criterion. We propose to use a length scale based on the vertical velocity, $\mathcal{L}=w_{\text {rms }} / N$, where $w_{\text {rms }}=\left\langle w^{2}\right\rangle^{1 / 2}$. A fluid parcel can be displaced upwards by a distance that is a maximum when all of the kinetic energy of its vertical motion, taken as $(1 / 2) w_{\mathrm{rms}}^{2}$, is converted into potential energy, and this maximum displacement is given by $\mathcal{L}$ (see Ref. [27]). As such, this length scale is a measure of the largest overturning scale in stratified turbulence, making it a suitable demarcation scale between anisotropic and isotropic motions. The corresponding wave number is $\kappa=N / w_{\text {rms }}$ and we use $\kappa$ as the filter horizontal wave number.

In a recent theoretical study of stratified turbulence [28] it was found that the vertical velocity variance is $w_{\text {rms }}^{2} \sim(\mathrm{Fr}) u_{h}^{2}=\epsilon / N$, which has been confirmed for DNS of decaying stratified turbulence [8]. As a result, it is expected that the scaling for the wave number $\kappa$ is $\kappa=N / w_{\text {rms }} \sim N /(\epsilon / N)^{1 / 2}=\sqrt{N^{3} / \epsilon}=k_{\mathrm{oz}}$. However, numerically $\kappa$ and $k_{\mathrm{oz}}$ are likely clearly distinct. We prefer to choose $\mathcal{K}_{h}=\kappa$ because this wave number corresponds directly to the overturns and hence to the transition to isotropic motions in strongly stratified turbulence.

\section{NUMERICAL METHODS}

In this work DNS was performed in a numerical domain without solid boundaries with the purpose of simulating homogeneous stratified turbulence. We consider a linearly stratified fluid with constant Brunt-Väisälä frequency $N$ that is uniform throughout the numerical domain. In the DNS the turbulence is brought to an approximate steady state by introducing an artificial body force $\mathbf{f}$, which has been selected to inject energy only in the vortex component of the flow and not in the wave component directly. More details on the forcing technique used in the simulations are given in the Appendix. The equations being solved numerically are the Navier-Stokes equation with the Boussinesq approximation and the buoyancy equation:

$$
\begin{gathered}
\frac{\partial \mathbf{u}}{\partial t}+\mathbf{u} \cdot \nabla \mathbf{u}=-\frac{1}{\rho_{0}} \nabla p^{\prime}+v \nabla^{2} \mathbf{u}+b \mathbf{e}_{z}+\mathbf{f}, \\
\frac{\partial b}{\partial t}+\mathbf{u} \cdot \nabla b=\mathcal{D} \nabla^{2} b-N^{2} w
\end{gathered}
$$

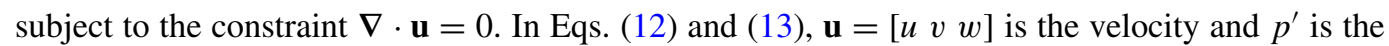
pressure perturbation away from hydrostatic pressure. There is no mean flow in the simulations, $\langle\mathbf{u}\rangle=0$, and so $\mathbf{u}$ represents the turbulence only. In addition to the Brunt-Väisälä frequency $N$, the input parameters to the DNS are the viscosity $v$ and the density diffusivity $\mathcal{D}$.

The numerical methodology consists of a pseudospectral method based on Rogallo's algorithm [29] in which Eqs. (12) and (13) are transformed into Fourier space. The boundary conditions are periodic in all three directions. The solution is advanced in time using a Runge-Kutta method of the second order and, during time integration, the velocity and buoyancy fields are multiplied by suitable integrating factors, allowing the viscous and diffusive terms in Eqs. (12) and (13) to 
TABLE I. List of DNS runs performed with important nondimensional parameters and values of the buoyancy and Ozmidov wave numbers. All values are obtained from time-averaged quantities, where the time averaging is over the statistically steady period of each DNS run. The naming convention for the runs is based on the Froude number: "F" followed by $\mathrm{Fr} \times 10^{2}$ (approximated to the first digit). The Reynolds number based on the Taylor microscale is defined as $\operatorname{Re}_{\lambda}=\left\langle u^{2}\right\rangle /\left(\nu\left\langle(\partial u / \partial x)^{2}\right\rangle^{1 / 2}\right)$.

\begin{tabular}{lccccccccc}
\hline \hline Name & $N_{x}$ & $N_{y}$ & \multicolumn{1}{c}{$N_{z}$} & $\mathrm{Fr}$ & $\operatorname{Re}_{b}$ & $\operatorname{Re}_{\lambda}$ & $L_{z} / \ell_{v}$ & \multicolumn{1}{c}{$k_{b}$} & $k_{\mathrm{oz}}$ \\
\hline F4 & 1024 & 1024 & 512 & 0.044 & 13.6 & 229 & 10.6 & 9.8 & 46.6 \\
F3 & 2048 & 2048 & 512 & 0.028 & 14.1 & 588 & 7.1 & 14.4 & 87.0 \\
F2 & 4096 & 4096 & 1024 & 0.021 & 17.0 & 818 & 8.9 & 22.1 & 153.8 \\
F1 & 8192 & 8192 & 2048 & 0.014 & 21.7 & 1377 & 14.6 & 30.1 & 258.4 \\
\hline \hline
\end{tabular}

be calculated exactly. The time step $\Delta t$ is calculated using an advection Courant-Friedrichs-Lewy (CFL) condition. The numerical domain is rectangular with horizontal dimensions $L_{x}=L_{y}=2 \pi$ and vertical dimension $L_{z}<L_{x}$ to reduce the computational cost of the DNS by taking advantage of the large-scale anisotropy of the flow. Tests were made at the lower grid resolution by varying the vertical dimensions of the domain and it was found that the results became relatively insensitive to the vertical dimension for $L_{z} / \ell_{v} \geqslant 7$. The exact value of $L_{z}$ for each simulation was then chosen after some trial and error to ensure that $L_{z} / \ell_{v} \geqslant 7$ so that there are at least seven turbulent layers in each simulation. Here $\ell_{v}$ is the vertical length scale obtained from the average of the integral length scales of $u$ and $v$ in the vertical direction. The number of grid points was chosen to satisfy $L_{z}=\left(N_{z} / N_{x}\right) L_{x}$ with $N_{x}=N_{y}$, where the numbers of grid points in the three directions are $N_{x}$, $N_{y}$, and $N_{z}$. This choice ensures that the grid spacing is the same in all three directions. The high-resolution run F1 was initialized from a snapshot of run F2 to reduce the number of time steps required to reach statistical stationarity and so it was necessary to make the conservative choice of maintaining the same aspect ratio of the previous run. At the largest scales, the first nonzero horizontal wave number is $k_{h}=1$ while the forcing is concentrated at $k_{h}=3$. The dealiasing technique consists of a combination of phase shifting and truncation. In the phase-shifting technique, random phase shifts are applied to the velocity and buoyancy fields in both steps of the time integration, which results in dealiasing of the new solution at the following time step (for more details see Ref. [29]). Considering that phase shifting is used, the truncation is active only for wave vectors with $k=|\mathbf{k}|>k_{\max }=(2 \sqrt{2} / 3) k_{\text {nyq }} \approx 0.94 k_{\text {nyq }}$, where $k_{\text {nyq }}=N_{x} / 2$ is the maximum wave number for which the physical solution is correctly captured.

In Table I details of the four DNS runs that were performed are listed including the important nondimensional parameters. We have carried out four DNS runs at successively higher resolution in order to decrease the Froude number gradually towards $\mathrm{Fr} \ll 1$ while keeping $\operatorname{Re}_{b}>10$ in all runs. All DNS runs have $k_{\max } \eta \approx 1.5$ during steady state, where $\eta=\left(v^{3} / \epsilon\right)^{1 / 4}$ is the Kolmogorov length scale, and this is considered a sufficient resolution of the dissipative scales for the present purposes (see Ref. [26]). The power input $P$ provided by the forcing is kept at the same value for all the simulations and this leads to a kinetic energy dissipation $\epsilon$ at steady state that does not vary significantly across the four runs. Hence, the viscosity is decreased as the grid resolution is increased from run F4 to run F1, in accordance with the above condition for the good resolution of the small scales. As a result, the Reynolds number based on the Taylor microscale increases from run $\mathrm{F} 4$ to run F1 to high values, $\operatorname{Re}_{\lambda}=O\left(10^{3}\right)$. Our aim was to keep the buoyancy Reynolds number at $\operatorname{Re}_{b} \approx 15$ for runs F4 to F2 since there is evidence that at $\mathrm{Re}_{b}=O(10)$ some of the important features of strongly stratified turbulence are observed $[8,11]$. The objective for the highest resolution simulation, run $\mathrm{F} 1$, was to increase this value slightly to $\mathrm{Re}_{b} \approx 22$. This means that run $\mathrm{F} 1$ satisfies the condition $\operatorname{Re}_{b}>20$, which was identified as the criterion to have active three-dimensional turbulence at small scales in stratified shear layers [27]. Since $\operatorname{Re}_{b}=\epsilon / \nu N^{2}$, this allowed $N$ to be increased from run F4 to run F1, resulting in a continuously decreasing Froude number, down to $\mathrm{Fr}=O\left(10^{-2}\right)$. The fact that Fr decreases from run F4 to run F1 can also be seen from the relation between $\operatorname{Re}_{b}$ and 


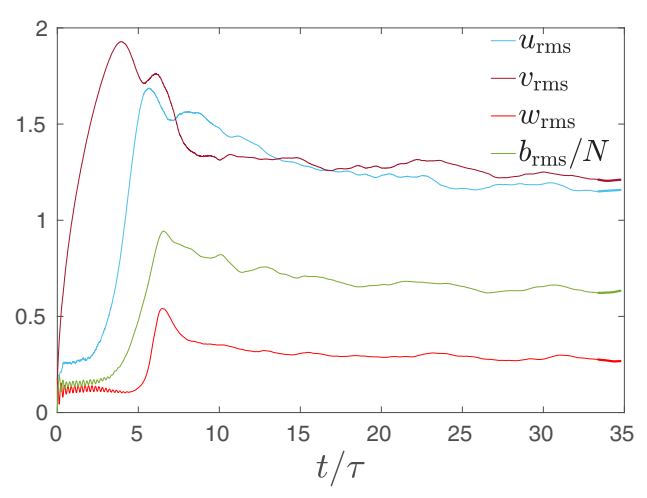

(a)

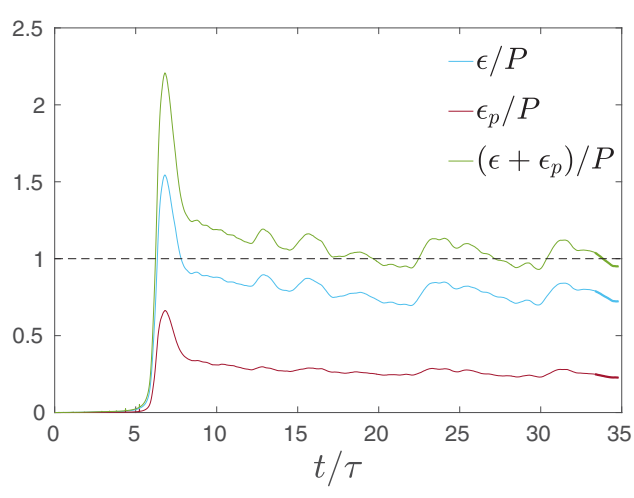

(b)

FIG. 3. Evolution of volume-averaged quantities for run F2: (a) root-mean-square velocity components and buoyancy normalized by $N$, and (b) kinetic energy dissipation, potential energy dissipation, and the total dissipation $\epsilon+\epsilon_{p}$, normalized by the input power $P$. The heavy portion of the curves, towards the end of the simulation, shows the period over which time averaging is performed.

Fr: $\operatorname{Re}_{b} \approx(1 / 15) \operatorname{Re}_{\lambda}^{2} \mathrm{Fr}^{2}$ (see Ref. [30]). Overall, these choices mean that runs F4 and F3 are in the transition region between weakly stratified and strongly stratified turbulence, while runs F2 and F1 are in the strongly stratified turbulence regime. This statement is based on experience and on the classification of the regimes in stratified turbulence in Fig. 18 of Ref. [2]. The Prandtl number in all the simulations is $\operatorname{Pr}=v / \mathcal{D}=1$.

\section{RESULTS}

\section{A. Time evolution}

In the DNS, the magnitude of the velocity field is increased from approximately zero through the use of forcing at the largest scales of the simulation and, after some time, the buoyancy field is also excited as kinetic energy gets converted into potential energy. This procedure was applied for all runs, except for run F1, which was initialized using a snapshot of run F2. The time evolution of run $\mathrm{F} 2$ is presented in terms of the root-mean-square values of the velocity components and of buoyancy and in terms of kinetic and potential energy dissipation rates in Fig. 3. We normalize time by the eddy-turnover time $\tau=\ell_{h} / u_{h}$, where $\ell_{h}$ is the horizontal length scale obtained from the average of the longitudinal integral length scales of $u$ and $v$, whereas $u_{h}=\left[(1 / 2)\left\langle u^{2}+v^{2}\right\rangle\right]^{1 / 2}$ is the horizontal velocity scale. As expected for strongly stratified turbulence, the vertical velocity is significantly smaller than the two horizontal velocity components as the stratification resists vertical motion, while the quantity $b_{\text {rms }} / N$ is about half of $u_{\text {rms }}$ and $v_{\text {rms }}$, showing that the potential energy is smaller but of the same order of magnitude compared to the kinetic energy. It is clear from Fig. 3 that after an initial transient all quantities reach a steady state. For run F2, the steady state is reached at about $t / \tau \approx 25$ but the time averaging of these quantities and of vertical spectra is performed only over the last $\approx 1.5 \tau$ of the simulation. It was found that both volume-averaged quantities and energy spectra are relatively insensitive to making the time-averaging period longer. The forcing technique used allows the input power to be controlled and kept to a constant value $P$ at each time step. Therefore, at steady state we should have $\epsilon+\epsilon_{p}=P$ and we can see that this physical condition is satisfied, with the quantity $\left(\epsilon+\epsilon_{p}\right) / P$ oscillating around a value of unity after the initial transient.

\section{B. Vertical spectra}

Let us now consider the vertical spectra in the simulations during steady state. The Froude number was gradually decreased across the four DNS runs with the objective of increasing the width 


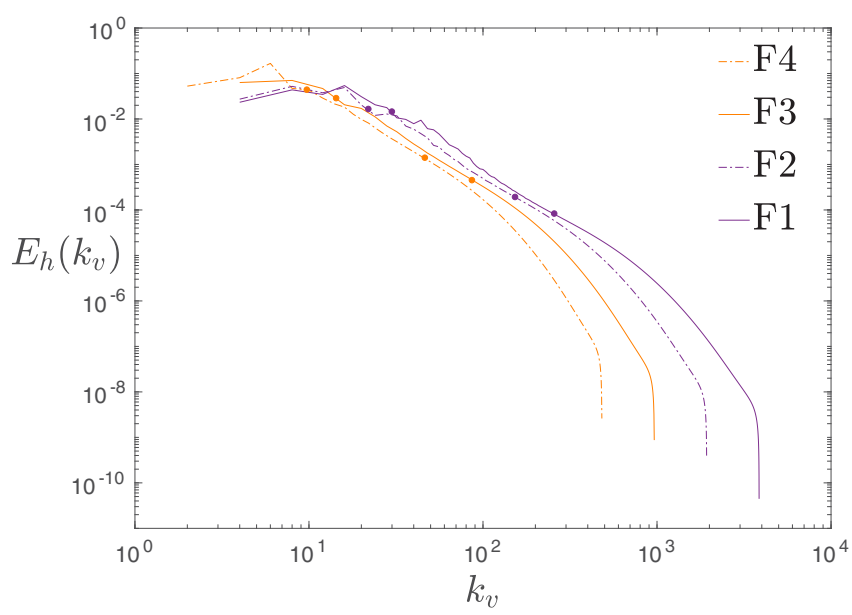

FIG. 4. Vertical spectra of horizontal kinetic energy time averaged during steady state. The solid circles superimposed on the curves indicate $k_{b}$ and $k_{\mathrm{oz}}$.

of the buoyancy-inertial range, which scales as $\mathrm{Fr}^{-1 / 2}$. Hence, for run $\mathrm{F} 1$ with $\mathrm{Fr}=0.014$ there is about a decade in Fourier space between the buoyancy and Ozmidov wave numbers over which the buoyancy-inertial range should occur. The time-averaged vertical energy spectra are shown in Fig. 4. The vertical spectra are relatively steep and have a similar behavior at varying Froude number, extending to higher vertical wave numbers from run F4 to run F1 as the resolution is increased and the dissipation scales become smaller. There appears to be a bump and a deviation from a power-law behavior in the vertical spectra, which is visible at wave numbers higher than the Ozmidov wave number. To investigate this further and in particular to look for a buoyancy-inertial range, we plot the vertical spectra of horizontal kinetic energy and of potential energy normalized by $N^{2} k_{v}^{-3}$ in Fig. 5 . These compensated forms clearly show the bump in the vertical spectra at high wave numbers and make it evident that the vertical spectra are in disagreement with $E_{h}\left(k_{v}\right) \propto E_{p}\left(k_{v}\right) \propto N^{2} k_{v}^{-3}$. The present results are similar to what was previously observed in simulations of stratified turbulence $[8,11,12,22]$. For the two DNS runs with the lowest Froude number there is a narrow constant plateau in the compensated form of $E_{h}\left(k_{v}\right)$ - this occurs only for run F1 in the case of $E_{p}\left(k_{v}\right)$-at

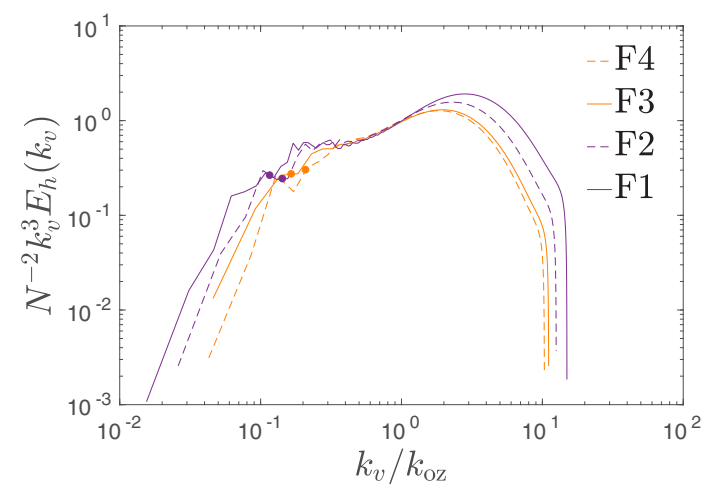

(a)

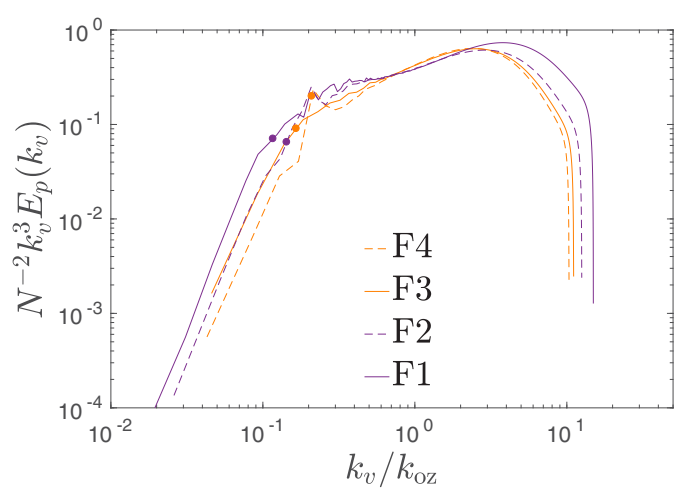

(b)

FIG. 5. Vertical spectra in compensated form of (a) kinetic energy and (b) potential energy. The solid circles indicate the buoyancy wave number. 


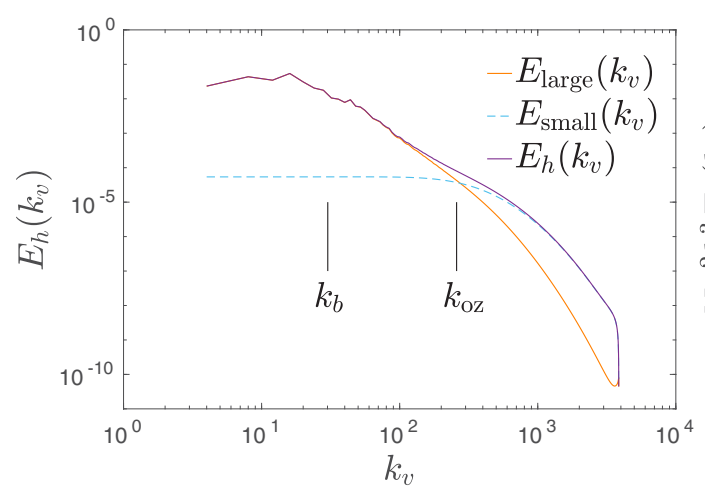

(a)

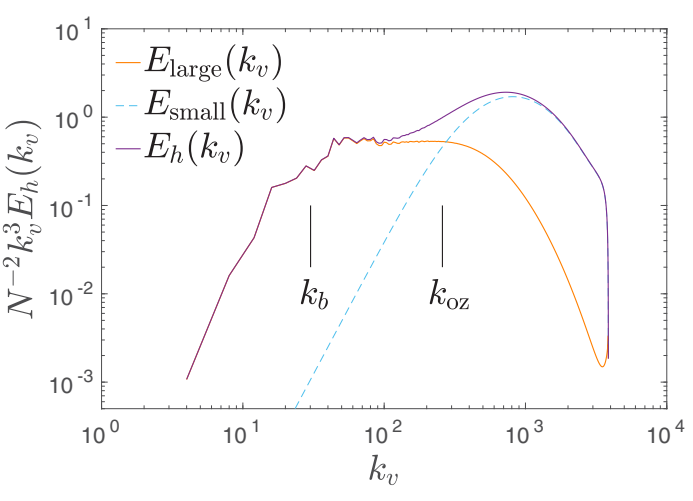

(b)

FIG. 6. Vertical spectra in run F1 using the scale decomposition: large scale, small scale, and full vertical spectra, (a) true form and (b) compensated form.

vertical wave numbers slightly higher than the buoyancy wave number. This is suggestive of a buoyancy-inertial range starting to become visible at $\mathrm{Fr} \approx 0.02$ and lower. Overall, however, the discrepancy with the strongly stratified turbulence theory remains since the buoyancy-inertial range should extend up to the Ozmidov wave number. As shown in Fig. 5, at $k_{v}=k_{\mathrm{oz}}$ and at higher wave numbers there is an excess of kinetic and of potential energy compared to what is expected from theory.

\section{Large-scale vertical spectra}

We calculate large-scale vertical spectra according to Eqs. (8) and (10) from results of the DNS. In Fig. 6(a) the results of the scale decomposition of the vertical energy spectrum for run F1 are presented. The large-scale vertical spectrum $E_{\text {large }}\left(k_{v}\right)$ follows the full vertical spectrum closely at low wave numbers, where the small-scale vertical spectrum $E_{\text {small }}\left(k_{v}\right)$ is flat and of low magnitude. At $k_{v} \approx 100$ the large-scale vertical spectrum starts to diverge and become steeper than the full spectrum as a significant portion of the kinetic energy at this and at higher wave numbers is contained in the small-scale spectrum. Eventually $E_{\text {large }}\left(k_{v}\right)$ and $E_{\text {small }}\left(k_{v}\right)$ cross over at $k_{v} \approx k_{\mathrm{oz}}$, and at higher wave numbers the small-scale vertical spectrum dominates. The large-scale and small-scale vertical spectra presented in Fig. 6 and in the remaining part of this paper were calculated by computing the wave number $\kappa=N / w_{\text {rms }}$ at a given time instant and then using $\mathcal{K}_{h}=\kappa$ to calculate the large-scale and small-scale vertical spectra at this time instant. This process was repeated for several time instants during steady state using the instantaneous value of $\kappa$ at each of these time instants for the filtering, and hence a set of large-scale and small-scale vertical spectra were obtained, which were subsequently time averaged. For consistency, the time-averaging period was chosen to be the same as for the other quantities of interest (see Fig. 3).

In Fig. 6(b) the compensated form of the large-scale vertical spectrum in run F1 is given. From this plot we can see that $E_{\text {large }}\left(k_{v}\right) \propto k_{v}^{-3}$ convincingly over a range of vertical wave numbers, from a wave number a little higher than $k_{b}$ to a wave number close to $k_{\mathrm{oz}}$. This is in agreement with a buoyancy-inertial range over this range of vertical wave numbers and at low horizontal wave numbers $k_{h} \leqslant \kappa$. The compensated form of $E_{\text {small }}\left(k_{v}\right)$ highlights that the bump in $E_{h}\left(k_{v}\right)$ is caused by small-scale motions with $k_{h}>\kappa$, which effectively cover up the $k_{v}^{-3}$ range in the full vertical spectrum. This result does not yet confirm that $E_{\text {large }}\left(k_{v}\right) \propto N^{2} k_{v}^{-3}$ as we still have to verify the proportionality with $N^{2}$, the background buoyancy gradient. This can be addressed by turning to the large-scale vertical spectra across the four DNS runs, which have varying background buoyancy gradient. In Fig. 7 the compensated form of $E_{\text {large }}\left(k_{v}\right)$ is shown as obtained from the four DNS 


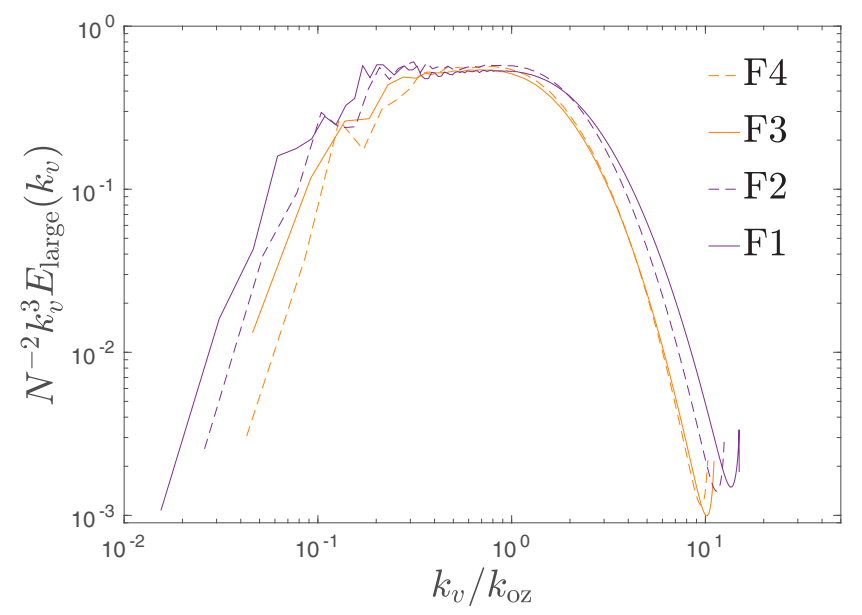

FIG. 7. Large-scale vertical spectra from the four DNS runs in compensated form.

runs. This confirms the proportionality of $E_{\text {large }}\left(k_{v}\right)$ with $N^{2}$ since there is a good collapse of the compensated spectra up to $k_{v} \approx k_{\mathrm{oz}}$, where the spectra diverge. It can be observed that the plateau gets wider from run F4 to run F1, as expected since the Froude number is decreased and so the separation between $k_{b}$ and $k_{\mathrm{oz}}$ gets larger. The range of validity of $E_{\text {large }}\left(k_{v}\right) \propto N^{2} k_{v}^{-3}$ is approximately $k_{b} \leqslant k_{v} \leqslant k_{\mathrm{oz}}$, perhaps with a scaling factor of order unity included in front of $k_{b}$. It is of interest to consider the value of the constant of proportionality, $\alpha$, of $E_{\text {large }}\left(k_{v}\right) \propto N^{2} k_{v}^{-3}$ in line with Eq. (1). This is estimated as $\alpha \approx 0.53$ from the results of run F1, which has the clearest buoyancy-inertial range. As can be seen in Fig 7, there is some variation of this value across the DNS runs and so there is a degree of uncertainty in the precise value of $\alpha$.

We now turn our attention to the large-scale vertical spectrum of potential energy $E_{p \text {,large }}\left(k_{v}\right)$. The compensated form of $E_{p \text {,large }}\left(k_{v}\right)$ for the four DNS runs is presented in Fig. 8. There is a constant plateau with a collapse for runs F1 and F2 in agreement with $E_{p, \text { large }}\left(k_{v}\right) \propto N^{2} k_{v}^{-3}$. On the other hand, there is not a clear plateau in runs F3 and F4, at higher Froude number. Considering runs $\mathrm{F} 1$ and F2 only, the plateau is narrower than that observed for the kinetic energy $\operatorname{spectra} E_{\text {large }}\left(k_{v}\right)$,

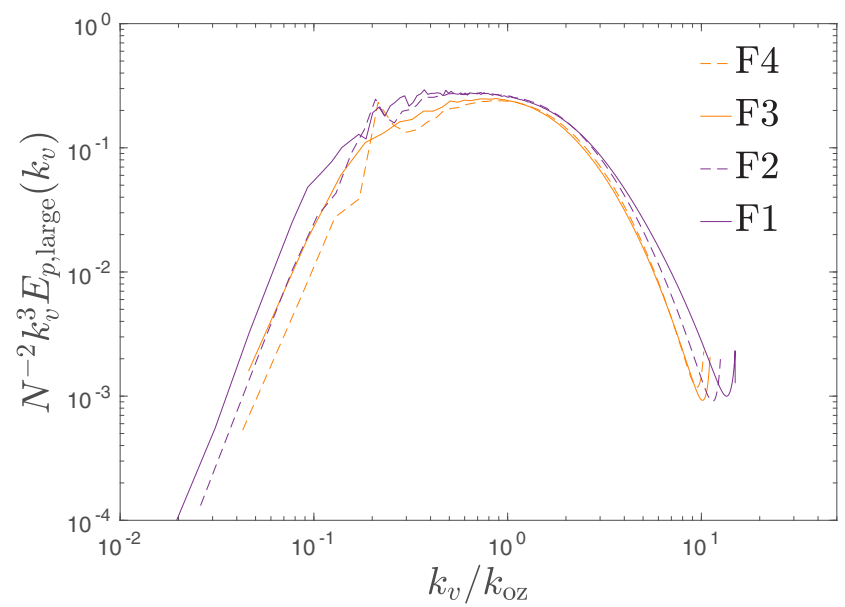

FIG. 8. Large-scale vertical spectra of potential energy in compensated form. 


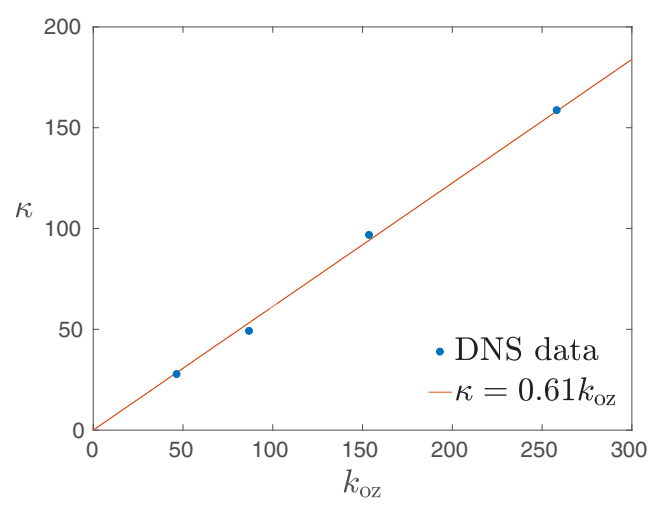

(a)

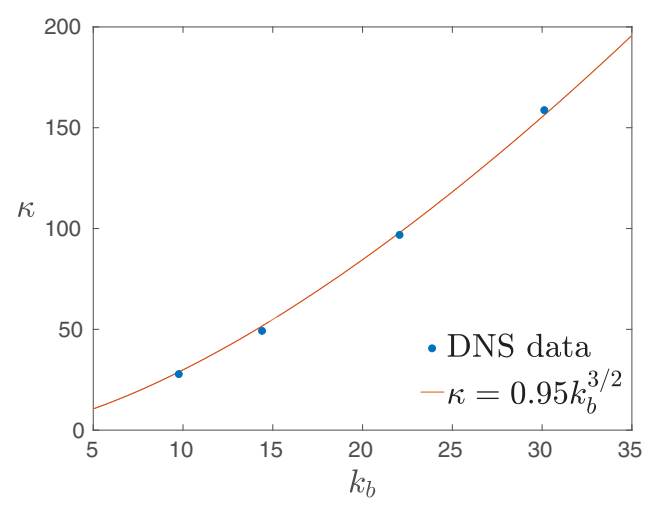

(b)

FIG. 9. Horizontal wave number $\kappa$ as a function of (a) the Ozmidov wave number $k_{\mathrm{oz}}$ and (b) the buoyancy wave number $k_{b}$. All wave numbers are presented as time-averaged values: $\kappa=N / \bar{w}_{\text {rms }}, k_{\mathrm{oz}}=\sqrt{N^{3} / \bar{\epsilon}}$, and $k_{b}=N / \bar{u}_{h}$. Each point corresponds to one of the four DNS runs.

starting from wave numbers considerably higher than $k_{v}=k_{b}$ and ending at $k_{v} \approx k_{\mathrm{oz}}$. The constant of proportionality as estimated from $E_{p \text {, large }}\left(k_{v}\right)$ of run $\mathrm{F} 1$ is $\beta \approx 0.27$. It is not well understood why the $k_{v}^{-3}$ range of the large-scale vertical spectra of potential energy starts at a wave number higher than $k_{b}$ and why it is narrower than the corresponding range in the large-scale vertical spectra of kinetic energy. The behavior of the corresponding fields in real space is in line with this behavior. Indeed, the vertical integral length scale $\ell_{v}$ obtained from the horizontal components of velocity is significantly larger than the integral length scale $\ell_{v, b b}$ obtained from the buoyancy field, consistently throughout the DNS runs. For example, for run F1 the ratio of their time-averaged values is $\ell_{v} / \ell_{v, b b}=4$.6. This means that the turbulent layers observed in the buoyancy field are thinner compared to the layers in the velocity field. Since $\ell_{v} \sim \ell_{b}$, the start of the $k_{v}^{-3}$ range, which should correspond to these integral length scales, is at $k_{v} \approx k_{b}$ for $E_{\text {large }}\left(k_{v}\right)$ and at a wave number $k_{v}>k_{b}$ for $E_{p \text {,large }}\left(k_{v}\right)$.

In summary, the results show that by using the scale decomposition the bump in the full vertical spectrum, created by small scales with $k_{h}>\kappa$, is filtered out. This uncovers the fact that there indeed is a range of scales in our DNS of strongly stratified turbulence over which buoyancy and inertia are in balance and with a local Froude number of the order of unity. The buoyancy-inertial range is observed approximately over the range $k_{b} \leqslant k_{v} \leqslant k_{\mathrm{oz}}$, in agreement with the strongly stratified turbulence theory. Over a narrower range of vertical wave numbers $E_{p \text {, large }}\left(k_{v}\right) \propto N^{2} k_{v}^{-3}$, showing that at these scales there is also approximate equipartition between kinetic and potential energy.

\section{The filter wave number}

As mentioned previously, the results obtained from the scale decomposition of the vertical spectrum are highly dependent on the particular filter wave number that we have chosen. Since the large-scale vertical spectra give results in agreement with our expectations and with theory, the filter horizontal wave number $\mathcal{K}_{h}=\kappa$ appears to provide a good demarcation between anisotropic scales and isotropic scales in stratified turbulence. It is therefore worth investigating the values of $\kappa$ across the different DNS runs. We have seen in Sec. II that the theoretical prediction is that $\kappa$ scales as the Ozmidov wave number. In Fig. 9, the time-averaged values of $\kappa$ in the four DNS runs are plotted both as a function of the Ozmidov wave number and of the buoyancy wave number. As can be seen in Fig. 9(a), there is a trend very close to $\kappa \propto k_{\mathrm{oz}}$ in the DNS runs, in agreement with theory. On the other hand, it is clear from Fig. 9(b) that $\kappa$ is not proportional to $k_{b}$ and that, as the buoyancy wave number is increased from run F4 to run F1 due to the increase in stratification, the wave number $\kappa$ increases significantly faster, according to $\kappa \propto k_{b}^{3 / 2}$. This is a reassuring result since this power law can be 


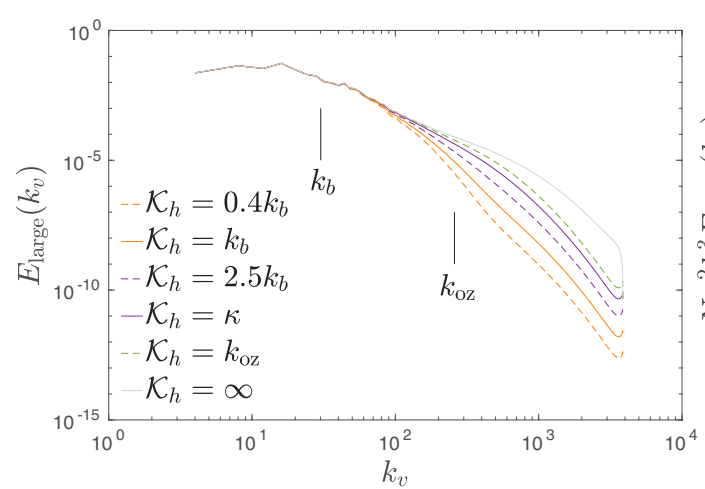

(a)

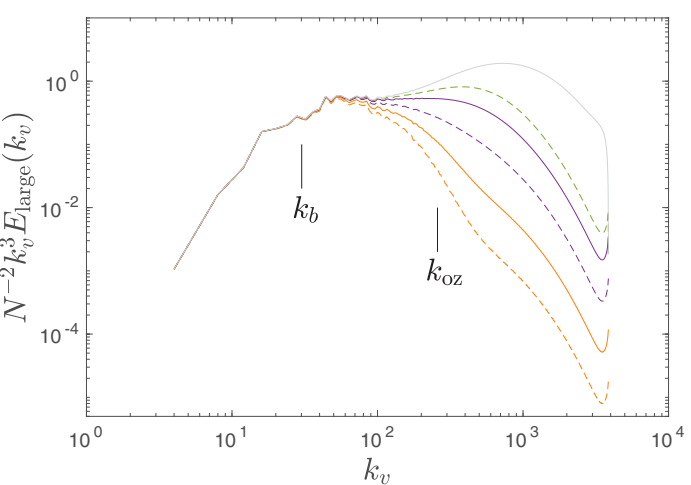

(b)

FIG. 10. Large-scale vertical spectra in run F1 obtained using different values of filter horizontal wave number: (a) true form and (b) compensated form (see legend in (a) for both figures). The large-scale vertical spectrum with $\mathcal{K}_{h}=\infty$ is equal to the full vertical spectrum $E_{h}\left(k_{v}\right)$. The time-averaged values of the filter horizontal wave numbers that were used are $\mathcal{K}_{h}=\left[0.4 k_{b}, k_{b}, 2.5 k_{b}, \kappa, k_{\mathrm{oz}}\right]=[12.1,30.1,75.4,158.5,258.4]$.

derived directly using theoretical arguments, $\kappa \sim k_{\mathrm{oz}}=\left(N / u_{h}\right)^{3 / 2}\left(u_{h}^{3} / \epsilon\right)^{1 / 2} \sim k_{b}^{3 / 2}$, noticing that $u_{h}^{3} / \epsilon \sim \ell_{h} \sim 1 / k_{h, f}=$ const since the forcing wave number $k_{h, f}$ is constant across the simulations (in the four runs indeed $u_{h}^{3} / \epsilon \approx$ const with variations of less than $10 \%$ from the mean value across the runs). All in all, these results support the classical interpretation of the Ozmidov length scale being the demarcation scale between scales affected by buoyancy and scales that are largely unaffected by buoyancy. The results also suggest that the largest overturning scale, estimated as $\mathcal{L}=1 / \kappa$, is of the order of the Ozmidov length scale and not of the order of the buoyancy length scale, as recently hypothesized in a number of numerical studies of stratified turbulence [12,20]. Indeed $\mathcal{L}=w_{\text {rms }} / N$ is significantly smaller than $\ell_{b}=u_{h} / N$, since the vertical velocity is much smaller than the horizontal velocity in stratified turbulence.

\section{E. Sensitivity to the filter wave number}

As mentioned in Sec. I, Augier et al. [12] computed vertical spectra at large horizontal scales from the results of their simulations of stratified turbulence. A filter horizontal wave number $\mathcal{K}_{h}=0.4 k_{b}$ was used, corresponding to a relatively large scale, slightly larger than the buoyancy length scale $\ell_{b}$. The large-scale vertical spectra obtained in this way were considerably steeper than $k_{v}^{-3}$ (see Fig. 18 of Ref. [12]). Considering the qualitative difference in results for $E_{\text {large }}\left(k_{v}\right)$ between the present work and that of Augier et al., it is of interest to consider the sensitivity of $E_{\text {large }}\left(k_{v}\right)$ to variations in the filter wave number $\mathcal{K}_{h}$. In Fig. 10 a number of large-scale vertical spectra obtained from the results of run F1 using different values of $\mathcal{K}_{h}$, from $\mathcal{K}_{h}=0.4 k_{b}$ to $\mathcal{K}_{h}=k_{\mathrm{oz}}$, are shown. There are significant departures from $E_{\text {large }}\left(k_{v}\right) \propto N^{2} k_{v}^{-3}$ for all large-scale vertical spectra obtained using a filter wave number different from $\mathcal{K}_{h}=\kappa$, showing that the sensitivity of $E_{\text {large }}\left(k_{v}\right)$ to $\mathcal{K}_{h}$ is high. The compensated large-scale vertical spectra obtained with filter wave numbers $\mathcal{K}_{h}<\kappa$ depart from the theoretical form at a wave number a little higher than $k_{b}$ and then decay rapidly at larger wave numbers. In particular for $\mathcal{K}_{h}=0.4 k_{b}$ the large-scale vertical spectrum becomes significantly steeper than $k_{v}^{-3}$, in accordance with the results of Augier et al. This highlights that a considerable number of horizontal scales smaller than $\ell_{b}$ contribute to forming the buoyancy-inertial range. On the other hand, for $\mathcal{K}_{h}=k_{\mathrm{oz}}>\kappa$ there is a small bump at high wave numbers and the general behavior of $E_{\text {large }}\left(k_{v}\right)$ transitions towards the behavior of the full vertical spectrum. It is interesting to note that for all filter wave numbers the large-scale vertical spectra are practically identical at low wave numbers, $k_{v}<k_{b}$, as are their values in the neighborhood of $k_{v}=k_{b}$. This shows that 


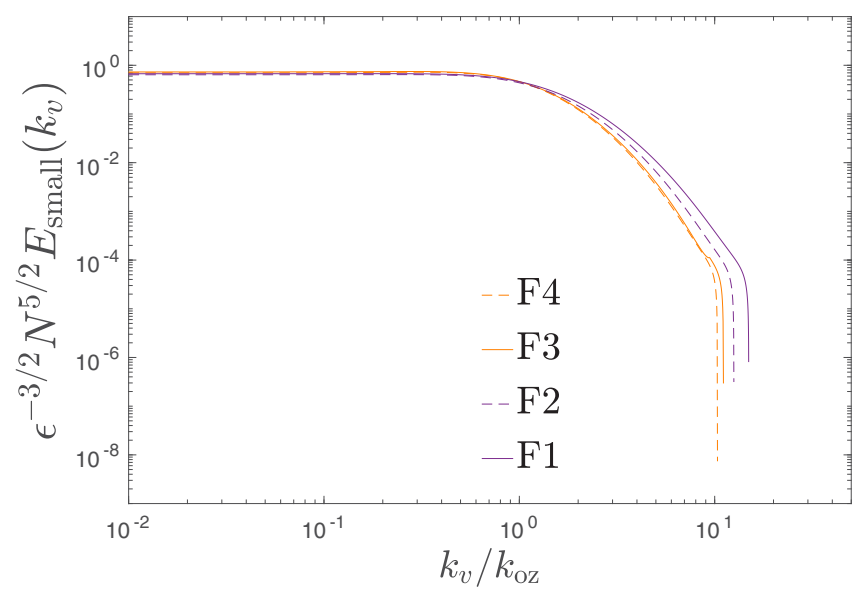

FIG. 11. Normalized small-scale vertical spectra. In order to show $E_{\text {small }}(0)$ this point has been plotted for all curves at $k_{v} / k_{\mathrm{oz}}=0.01$.

the kinetic energy in the largest vertical scales, corresponding to $k_{v} \sim k_{b}$, is mainly due to large horizontal scales with $k_{h}<0.4 k_{b}$, and that the contribution from horizontal wave numbers beyond this value is negligible. This agrees with the picture of energetic layers in stratified turbulence at the largest scales.

\section{F. Small-scale vertical spectra}

In this section we consider the small-scale vertical spectra and their scaling laws. Plots of $E_{\text {small }}\left(k_{v}\right)$ normalized using $\epsilon$ and $N$ are given in Fig. 11. The small-scale vertical spectra achieve their maximum value over a relatively flat region up to $k_{v} \approx k_{\mathrm{oz}}$ for all runs. This would appear to suggest that structures with small horizontal scales and large vertical scales carry considerable kinetic energy, which would be an unexpected result. However, this is not the case. The reason why $E_{\text {small }}\left(k_{v}\right)$ does not decay to zero at small $k_{v}$ as may be expected is that $E_{\text {small }}(0)$ is related to the vertical length scale of the velocity field at small horizontal scales, $\mathbf{u}_{\text {small }} \mathcal{H}(\mathbf{x})$, defined conversely to $\mathbf{u}_{\text {large } \mathcal{H}}(\mathbf{x})$ given in Eq. (7). This is because the one-dimensional (1D) energy spectrum $E_{\text {small }}\left(k_{v}\right)$ is the Fourier transform of the correlation $R_{\text {small }}\left(r_{z}\right)=\left\langle u_{\text {small } \mathcal{H}}(\mathbf{x}) u_{\text {small } \mathcal{H}}\left(\mathbf{x}+r_{z} \mathbf{e}_{z}\right)\right\rangle+$ $\left\langle v_{\text {small } \mathcal{H}}(\mathbf{x}) v_{\text {small } \mathcal{H}}\left(\mathbf{x}+r_{z} \mathbf{e}_{z}\right)\right\rangle$. It follows that $E_{\text {small }}(0)=1 / \pi\left(\left\langle u_{\text {small } \mathcal{H}}^{2}\right\rangle l_{11,3}+\left\langle v_{\text {small } \mathcal{H}}^{2}\right\rangle l_{22,3}\right)$, where $l_{11,3}$ and $l_{22,3}$ are the integral length scales in the vertical direction obtained from the two terms in $R_{\text {small }}\left(r_{z}\right)$. These quantities are nonzero and so $E_{\text {small }}(0)$ is finite. In addition, for an isotropic flow field the 1D transverse energy spectrum $E_{22}\left(k_{1}\right)$ has a maximum at $k_{1}=0$ (see Sec. 6.5 of Ref. [26]). Of course $\mathbf{u}_{\text {small } \mathcal{H}}(\mathbf{x})$ is not strictly isotropic, yet it appears that $E_{\text {small }}\left(k_{v}\right)$ is relatively flat from $k_{v}=0$ to $k_{v} \approx k_{\mathrm{oz}}$, beyond which it decays. The flat portion of $E_{\text {small }}\left(k_{v}\right)$ shows that most of the kinetic energy of the small-scale velocity field, related to $k_{v} E_{\text {small }}\left(k_{v}\right)$, is actually concentrated at $k_{v} \approx k_{\mathrm{oz}}$. This fact is confirmed by the good collapse of the small-scale vertical spectra at low wave numbers when these are normalized by $\epsilon^{3 / 2} N^{-5 / 2}=u_{\mathrm{oz}}^{2} \ell_{\mathrm{oz}}$, where $u_{\mathrm{oz}}=\sqrt{\epsilon / N}$ is the velocity of turbulent structures at the Ozmidov scale. This means that $E_{\text {small }}(0) \sim u_{\mathrm{oz}}^{2} \ell_{\mathrm{oz}}$, which suggests that $\left\langle\left|\mathbf{u}_{\text {small } \mathcal{H}}\right|^{2}\right\rangle \sim u_{\mathrm{oz}}^{2}$ and $l_{11,3} \sim l_{22,3} \sim \ell_{\mathrm{oz}}$.

According to the strongly stratified turbulence theory the vertical spectra should present classical inertial ranges at small scales: a Kolmogorov form $E_{3 \mathrm{D}}\left(k_{v}\right)=\epsilon^{2 / 3} k_{v}^{-5 / 3}$ for the kinetic energy spectrum and a form valid for passive scalar advection, $E_{p, 3 \mathrm{D}}=\left(\epsilon_{p} / \epsilon_{k}^{1 / 3}\right) k_{v}^{-5 / 3}$, for the potential energy spectrum. These forms may be presented by the small-scale vertical spectra and this is investigated in Fig. 12, which shows $E_{\text {small }}\left(k_{v}\right)$ and $E_{p \text {,small }}\left(k_{v}\right)$ compensated according to the above spectra. There are no inertial ranges in $E_{\text {small }}\left(k_{v}\right)$ and $E_{p \text {,small }}\left(k_{v}\right)$. This is probably due to the fact 


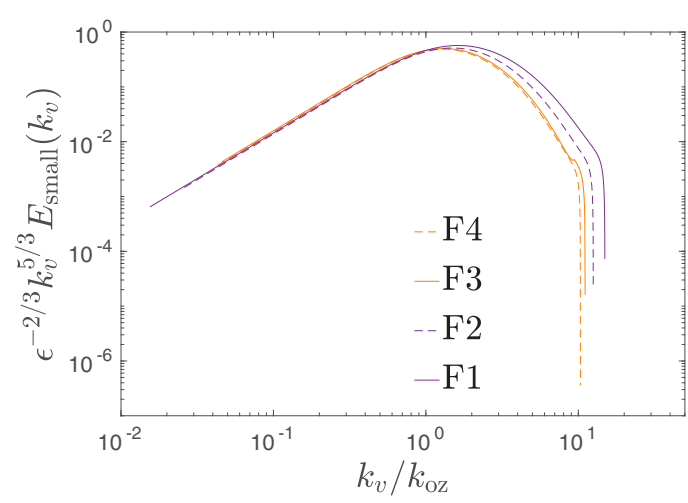

(a)

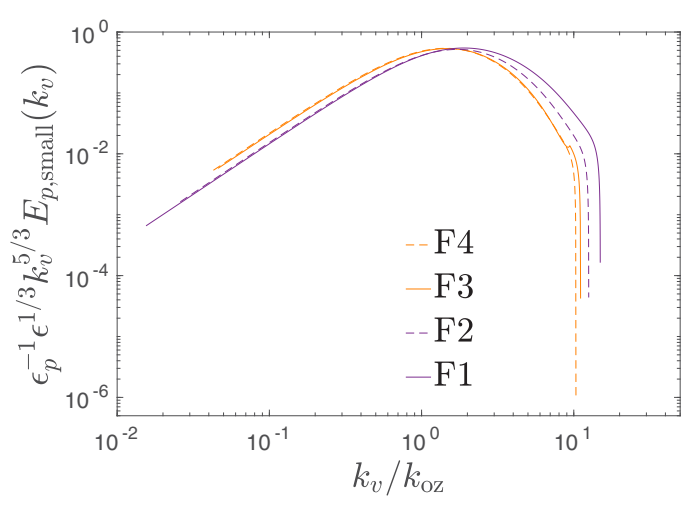

(b)

FIG. 12. Small-scale vertical spectra of (a) kinetic energy and (b) potential energy, compensated according to the isotropic forms.

that the buoyancy Reynolds number is not very large in the DNS, $\operatorname{Re}_{b}=O(10)$, and so there are not sufficient scales beyond the Ozmidov scale for an inertial range behavior to develop. The peaks in the compensated forms are all approximately at the same value close to unity, especially in the case of $E_{p \text {,small }}\left(k_{v}\right)$. Whether this is a hint of what will be an inertial range at higher $\mathrm{Re}_{b}$ or just a feature of the present small-scale spectra remains to be determined. As a final point, we remark that in the previous scale decomposition of vertical spectra of Ref. [23], the small-scale vertical spectra exhibit an inertial range, beyond $k_{v}=k_{\mathrm{oz}}$. The discrepancy with our results could be due to two reasons: the use of $k_{b}$ as the filter wave number, thus increasing the number of horizontal scales contributing to $E_{\text {small }}\left(k_{v}\right)$ compared to the present study, and the use of hyperviscosity in the simulations [23], which increases the range of wave numbers in $E_{\text {small }}\left(k_{v}\right)$ that could take part in an inertial range.

\section{DISCUSSION}

We have presented results for the large-scale vertical spectra that agree with $E_{\text {large }}\left(k_{v}\right) \propto N^{2} k_{v}^{-3}$ over a range of vertical wave numbers. Considering that this result holds at large horizontal scales, the fact that the full vertical spectrum $E_{h}\left(k_{v}\right)$ does not agree with this form as seen in the present DNS and in several previous studies $[8,11,13,22,23]$ should be due to the use of all horizontal scales in computing $E_{h}\left(k_{v}\right)$, including scales that are approximately isotropic. For these small scales there is not a buoyancy-inertial balance as buoyancy effects are negligible at these scales. Our results show that these small horizontal scales are responsible for the bump in the vertical spectrum at high wave numbers, whose effect is noticeable at much lower wave numbers down to the buoyancy wave number in simulations at moderately low Fr. This excess in kinetic energy effectively overshadows the buoyancy-inertial range in $E_{h}\left(k_{v}\right)$. This brings us to believe that the discrepancy observed thus far between experiments and simulations compared to the strongly stratified turbulence theory is in large part due to the aliasing problem associated with one-dimensional vertical energy spectra. Now, making an extrapolation to the case of very high $\mathrm{Re}_{b}$, it is expected that the contributions from small-scale turbulence will be distributed over a high number of scales so that the effect on $E_{h}\left(k_{v}\right)$ at low $k_{v}$ will become smaller. At the same time, in the limit of very low Fr the buoyancy-inertial range will be very wide. Therefore, considering these effects together, in the limit of strongly stratified turbulence a clear buoyancy-inertial range should be observed in the full vertical spectrum $E_{h}\left(k_{v}\right)$, without the need to perform a scale decomposition of the turbulent fields, and there is a hint of this behavior in our DNS with the lowest Froude number. It is likely for this reason that a buoyancy-inertial range is routinely observed in the full vertical spectrum in the atmosphere and in the oceans. 
In terms of the filter wave number, we have seen previously that it is proportional to $k_{\mathrm{oz}}$, meaning that the Ozmidov length scale is indeed a good demarcation scale between anisotropic and approximately isotropic motions. There is a wider significance to this result that may be of practical interest. In recent years, studies have been made of stratified turbulence using large eddy simulation (LES) [31,32], which is a promising approach for problems such as ocean turbulence considering the vast range of scales involved. In these LES studies isotropic subgrid-scale models are employed [31,32]. The present results suggest the use of a grid spacing $\Delta \approx(1 / \kappa)$ or $\Delta \approx \ell_{\mathrm{oz}}$ in LES, which would allow the anisotropic scales of motion to be captured in the simulation while modeling the isotropic scales occurring at smaller scales. However, in a recent LES study the grid spacing was chosen based on the buoyancy length scale and therefore a significantly larger scale than the Ozmidov length scale was used [32]. This does not seem appropriate since it means a considerable number of anisotropic scales are unresolved and it is likely that they will not be modeled correctly by an isotropic subgrid-scale model, which could explain the only partial match of the LES results with predictions for the strongly stratified turbulence regime [32]. It is important to underline that, even if a grid spacing $\Delta \approx \ell_{\mathrm{oz}}$ is chosen, the reduction in computational cost compared to an equivalent DNS is still very large. A representative buoyancy Reynolds number in the ocean is of the order of $\mathrm{Re}_{b}=100$ in regions of active turbulence [33]. If one stops computing at the Ozmidov length scale in a LES rather than at the Kolmogorov length scale, as is required in a DNS, the reduction in computational cost is of the order of $\left(\ell_{\mathrm{oz}} / \eta\right)^{3}=\mathrm{Re}_{b}^{9 / 4} \approx 3 \times 10^{4}$, which is a very significant reduction.

The vertical spectrum in the buoyancy-inertial range given in Eq. (1) was first proposed in relation to measurements of turbulence in the atmosphere [14,15] and in the oceans $[16,17]$. In the case of ocean turbulence, clear measurements of vertical shear spectra in agreement with $S\left(k_{v}\right) \approx N^{2} k_{v}^{-1}$ were made at vertical scales from $s_{v}=10 \mathrm{~m}$ down to $s_{v}=1 \mathrm{~m}$ by Gargett et al. [17], as shown in Fig. 1. In their measurements the Ozmidov length scale was close to the lower end of this range [17]. These observations are consistent with vertical energy spectra given by $E_{h}\left(k_{v}\right) \approx \alpha N^{2} k_{v}^{-3}$ up to $k_{v} \approx k_{\mathrm{oz}}$ and with $\alpha \approx 0.5$. The present DNS results for the large-scale vertical spectra also show a buoyancy-inertial range up to the Ozmidov wave number and give $\alpha \approx 0.53$, in good agreement with the ocean turbulence measurements. This positive match brings considerable support to the claim that strongly stratified turbulence with constant $N$ is a good model for turbulence in the ocean pycnocline. It would be of interest to compare also the value of $\beta$ obtained from the large-scale vertical spectra of potential energy with ocean measurements but this is not possible as most of the results in the literature concern vertical spectra of temperature variance (see, e.g., Ref. [16]) and to the best of our knowledge there are no measurements of vertical spectra of buoyancy variance where salinity also plays a role. The results for the kinetic energy spectra also bring about the question: Is $\alpha$ a universal constant in strongly stratified turbulence, independent of forcing technique, initial conditions, and mean flow? It is believed that this is the case, although considerably more evidence will have to be accumulated to demonstrate this hypothesis. What is definitely less clear is whether the value of $\alpha$ is in some way predictable, by recurring, for example, to arguments related to the condition for shear instability based on the Richardson number $\mathrm{Ri}$-namely, that $\mathrm{Ri}<1 / 4$-as attempted by Dewan [15]. This is an open question requiring further investigation.

\section{CONCLUSIONS}

In this paper, results from DNS of strongly stratified turbulence with forcing are analyzed using a scale decomposition of the turbulent flow and of the vertical energy spectra into large scales and small scales. In this way we have been able to recover large-scale vertical spectra $E_{\text {large }}\left(k_{v}\right) \propto N^{2} k_{v}^{-3}$ over a range of vertical wave numbers up to $k_{v} \approx k_{\mathrm{oz}}$. Also the large-scale vertical spectra of potential energy show $E_{p \text {,large }}\left(k_{v}\right) \propto N^{2} k_{v}^{-3}$ but over a narrower range of wave numbers. The present results constitute one of the first pieces of evidence coming from either experiments or numerical simulations clearly showing vertical spectra of this form, which have often been observed in the ocean and in the atmosphere. Moreover, they provide support for the existence of a range of scales in strongly 
stratified turbulence in which there is an approximate balance between buoyancy and inertia, in agreement with the strongly stratified turbulence theory. However, they also show that there are small-scale motions that are not in buoyancy-inertial balance and that result in the full vertical energy spectrum $E_{h}\left(k_{v}\right)$ not showing a buoyancy-inertial range in experiments and simulations at moderately low Froude number. This work highlights the importance of knowing both the horizontal wave number $k_{h}$ and vertical wave number $k_{v}$ of a structure in stratified turbulence to be able to characterize it correctly. In this highly anisotropic turbulent flow, descriptions using a single wave number inevitably have missing information, as is the case for the widely used one-dimensional energy spectra.

\section{ACKNOWLEDGMENTS}

I am grateful to E. Lindborg for interesting and stimulating discussions on stratified turbulence and for his suggestions that helped improve this manuscript. I am also thankful to G. Lupo and M. Atzori for discussions at an early stage of the present study. I acknowledge PRACE for the computational award StratForce "Direct numerical simulation of forced stratified turbulence" that allowed the present simulations to be carried out at CINECA, Italy. Computational resources at PDC in Sweden were made available by SNIC, which is gratefully acknowledged.

\section{APPENDIX: FORCING TECHNIQUE}

In defining the artificial body force in Fourier space we make use of the Craya-Herring reference frame, which has unit vectors given by (see, e.g., Ref. [6])

$$
\begin{gathered}
\mathbf{e}_{1}=\frac{\mathbf{k} \times \mathbf{e}_{z}}{\left|\mathbf{k} \times \mathbf{e}_{z}\right|}=\frac{k_{y}}{k_{h}} \mathbf{e}_{x}-\frac{k_{x}}{k_{h}} \mathbf{e}_{y}, \\
\mathbf{e}_{2}=\frac{\mathbf{k} \times \mathbf{e}_{1}}{\left|\mathbf{k} \times \mathbf{e}_{1}\right|}=\frac{k_{x} k_{z}}{k_{h} k} \mathbf{e}_{x}+\frac{k_{y} k_{z}}{k_{h} k} \mathbf{e}_{y}-\frac{k_{h}}{k} \mathbf{e}_{z}, \\
\mathbf{e}_{3}=\frac{\mathbf{k}}{|\mathbf{k}|}=\frac{k_{x}}{k} \mathbf{e}_{x}+\frac{k_{y}}{k} \mathbf{e}_{y}+\frac{k_{z}}{k} \mathbf{e}_{z} .
\end{gathered}
$$

The body force has to satisfy $\boldsymbol{\nabla} \cdot \mathbf{f}=0$ in order to generate a divergence-free velocity field. In Fourier space this means that $\mathbf{k} \cdot \hat{\mathbf{f}}=0$ and so the force can be written using only the first two components in the Craya-Herring frame, $\hat{\mathbf{f}}=\hat{f}_{1} \mathbf{e}_{1}+\hat{f}_{2} \mathbf{e}_{2}$. Similarly, the velocity field can be written as $\hat{\mathbf{u}}=\hat{u}_{1} \mathbf{e}_{1}+\hat{u}_{2} \mathbf{e}_{2}+\hat{\mathbf{u}}_{\mathrm{sm}}$. As can be seen from Eqs. (A1) and (A2), the first term, $\hat{u}_{1} \mathbf{e}_{1}$, corresponds to horizontal velocities that are vertically rotational, containing all the vertical vorticity $\omega_{z}=(\partial v / \partial x-\partial u / \partial y)$ of the flow. On the other hand, the second term $\hat{u}_{2} \mathbf{e}_{2}$ corresponds to three-dimensional velocities with $\omega_{z}=0$. The third term, $\hat{\mathbf{u}}_{\mathrm{sm}}$, represents the shear modes with $k_{x}=k_{y}=0$, which are modes that cannot be expressed in the Craya-Herring reference frame of Eqs. (A1)-(A3) and correspond to a mean flow in the horizontal direction that maintains a $z$ dependence. The classical interpretation is that the first term is the vortex component of the stratified flow and the second component is the wave component of the flow [3]. In terms of the forcing, if we choose a force that contains only the first term and set $\hat{f}_{2}=0$ the force will excite the vortex component of the velocity field only, as clearly shown in Ref. [12]. If we also choose to concentrate this vortical forcing in modes with $k_{z}=0$, this will generate vertically elongated vortices in the simulation that will undergo an instability possibly similar to the zigzag instability [34]. This instability will break the vertical coherence of the vortices, thereby generating internal gravity waves and injecting energy in the wave component of the flow as well. We have opted for vortical forcing with $k_{z}=0$ for three reasons: (i) to avoid exciting an arbitrary spectrum of internal gravity waves directly, (ii) to not predetermine the vertical length scale of the flow, and (iii) because this forcing was found to reduce the growth of energy in the shear modes $\mathbf{u}_{\mathrm{sm}}$ that would otherwise 
dominate the overall kinetic energy by the end of a simulation (as discussed in Ref. [30]). Vortical forcing with $k_{z}=0$ was used in several previous DNS studies of stratified turbulence [2,12,30].

In terms of the power spectrum, we choose $P\left(k_{h}\right)=\sum(1 / 2) \hat{f}_{1} \hat{f}_{1}^{*}=$ const, and this is concentrated at a horizontal wave number $k_{h}=3$. Hence, the following form of the forcing is used:

$$
\hat{f}_{1}= \begin{cases}\frac{c}{\sqrt{\pi k_{h}}} e^{i \theta} & \text { for } k_{h}=3 \\ 0 & \text { for } k_{h} \neq 3,\end{cases}
$$

where $c$ is a constant scaling factor, $i=\sqrt{-1}$, and $\theta$ is the random phase, uniformly distributed between $\theta=0$ and $\theta=2 \pi$. Note that the choice of domain dimensions $L_{x}=L_{y}=2 \pi$ means that the horizontal wave numbers in the simulation are discrete integers with spacing $\delta k=2 \pi / L_{x}=2 \pi / L_{y}=1$ for both $k_{x}$ and $k_{y}$. As a result, the wave vectors being forced are actually those lying in the band $2.5<k_{h}<3.5$ and with $k_{z}=0$. The phase $\theta$ is selected randomly for each wave vector at which forcing is applied and at each time step so that there is no time correlation of the forcing vectors. It is possible to calculate the power input of the forcing as

$$
P=\sum_{k_{x}, k_{y}, k_{z}} \hat{\mathbf{u}}^{*} \cdot \hat{\mathbf{f}}+\frac{1}{2} \hat{\mathbf{f}} \cdot \hat{\mathbf{f}}^{*} \Delta t=c P_{\mathrm{uf}}+c^{2} P_{\mathrm{ff}},
$$

where the first term is the "physical" power given by the scalar product of the velocity with the force, while the second term is the "artificial" power that exists only because of the discrete time steps of the simulation over which the force $\mathbf{f}$ is constant. In the final expression of Eq. (A5) the dependence of $P$ on the scaling factor $c$ has been made explicit. Now, we set the power $P$ to a certain value in our forcing method and ensure that the power provided is exactly $P$ by solving the quadratic equation for $c$ resulting from Eq. (A5), $P_{\mathrm{ff}} c^{2}+P_{\mathrm{uf}} c-P=0$, at every time step. This quadratic equation has the following solution for $c$ :

$$
c=\frac{-P_{\mathrm{uf}} \pm \sqrt{P_{\mathrm{uf}}^{2}+4 P_{\mathrm{ff}} P}}{2 P_{\mathrm{ff}}},
$$

and of the two roots we choose the one with minimum absolute value, which minimizes the magnitude of the force $\left|\hat{f}_{1}\right|$ while providing the given power $P$. We call this method "constant power minimal forcing"; it is an adaptation of a method proposed previously [35]. As can be seen by inspection of Eq. (A6), the choice of the value of $c$ with minimum absolute value means that the physical power input $P_{\text {phys }}=c P_{\text {uf }}>0$ so that the forcing always accelerates the flow on average. This choice is beneficial because it results in smooth time evolutions of rms velocity components and dissipation rates, as can be seen in Fig. 3. Indeed it was confirmed that other choices for $c$ give large oscillations of these quantities on a time scale $\Delta t$. Finally, constant power minimal forcing results in the artificial power input $P_{\text {artif }}=c^{2} P_{\mathrm{ff}} \ll P_{\text {phys }}$ in our DNS, with the time average of $P_{\text {artif }}$ being typically about $1 \%$ of the total input power.

[1] J. J. Riley, R. W. Metcalfe, and M. A. Weissman, Direct numerical simulations of homogeneous turbulence in density-stratified fluids, AIP Conf. Proc. 76, 79 (1981).

[2] G. Brethouwer, P. Billant, E. Lindborg, and J.-M. Chomaz, Scaling analysis and simulation of strongly stratified turbulent flows, J. Fluid Mech. 585, 343 (2007).

[3] J. J. Riley and M.-P. Lelong, Fluid motions in the presence of strong stable stratification, Annu. Rev. Fluid Mech. 32, 613 (2000).

[4] P. Billant and J.-M. Chomaz, Self-similarity of strongly stratified inviscid flows, Phys. Fluids 13, 1645 (2001).

[5] J. M. Holford and P. F. Linden, Turbulent mixing in a stratified fluid, Dyn. Atmos. Oceans 30, 173 (1999).

[6] F. S. Godeferd and C. Staquet, Statistical modelling and direct numerical simulations of decaying stably stratified turbulence. Part 2. Large-scale and small-scale anisotropy, J. Fluid Mech. 486, 115 (2003). 
[7] P. Augier, P. Billant, M. E. Negretti, and J.-M. Chomaz, Experimental study of stratified turbulence forced with columnar dipoles, Phys. Fluids 26, 046603 (2014).

[8] A. Maffioli and P. A. Davidson, Dynamics of stratified turbulence decaying from a high buoyancy Reynolds number, J. Fluid Mech. 786, 210 (2016).

[9] R. V. Ozmidov, On the turbulent exchange in a stably stratified ocean, Bull. (Izv.) Acad. Sci. USSR, Atmos. Ocean. Phys. 1, 493 (1965).

[10] E. Lindborg, The energy cascade in a strongly stratified fluid, J. Fluid Mech. 550, 207 (2006).

[11] P. Bartello and S. M. Tobias, Sensitivity of stratified turbulence to the buoyancy Reynolds number, J. Fluid Mech. 725, 1 (2013).

[12] P. Augier, P. Billant, and J.-M. Chomaz, Stratified turbulence forced with columnar dipoles: Numerical study, J. Fluid Mech. 769, 403 (2015).

[13] M. L. Waite and P. Bartello, Stratified turbulence generated by internal gravity waves, J. Fluid Mech. 546, 313 (2006).

[14] E. M. Dewan and R. E. Good, Saturation of "universal" spectrum for vertical profiles of horizontal scalar winds in the atmosphere, J. Geophys. Res. 91, 2742 (1986).

[15] E. Dewan, Saturated-cascade similitude spectra of gravity wave spectra, J. Geophys. Res. 102, 29799 (1997).

[16] M. C. Gregg, Variations in the intensity of small-scale mixing in the main thermocline, J. Phys. Ocean. 7, 436 (1977).

[17] A. E. Gargett, P. J. Hendricks, T. B. Sanford, T. R. Osborn, and A. J. Williams, A composite spectrum of vertical shear in the upper ocean, J. Phys. Oceanogr. 11, 1258 (1981).

[18] J. J. Riley and E. Lindborg, Stratified turbulence: A possible interpretation of some geophysical turbulence measurements, J. Atmos. Sci. 65, 2416 (2008).

[19] O. Praud, A. M. Fincham, and J. Sommeria, Decaying grid turbulence in a strongly stratified fluid, J. Fluid Mech. 522, 1 (2005).

[20] M. Waite, Stratified turbulence at the buoyancy scale, Phys. Fluids 23, 066602 (2011).

[21] P. Bartello (private communication).

[22] S. Almalkie and S. M. de Bruyn Kops, Kinetic energy dynamics in forced, homogeneous, and axisymmetric stably stratified turbulence, J. Turbul. 13, N29 (2012).

[23] P. Augier, J.-M. Chomaz, and P. Billant, Spectral analysis of the transition to turbulence from a dipole in stratified fluid, J. Fluid Mech. 713, 86 (2012).

[24] A. M. Obukhov, Spectral energy distribution in a turbulent flow, Izv. Akad. Nauk SSSR Ser. Geogr. Geofiz. 5, $453(1941)$.

[25] U. Frisch, Turbulence (Cambridge University Press, Cambridge, UK, 1995).

[26] S. B. Pope, Turbulent Flows (Cambridge University Press, Cambridge, UK, 2000).

[27] W. D. Smyth and J. N. Moum, Length scales of turbulence in stably stratified mixing layers, Phys. Fluids 12, 1327 (2000).

[28] J. J. Riley and E. Lindborg, Recent progress in stratified turbulence, in Ten Chapters in Turbulence, edited by P. A. Davidson, Y. Kaneda, and K. R. Sreenivasan (Cambridge University Press, Cambridge, UK, 2013).

[29] R. S. Rogallo, Numerical experiments in homogeneous turbulence, 81315, 1981.

[30] A. Maffioli, G. Brethouwer, and E. Lindborg, Mixing efficiency in stratified turbulence, J. Fluid Mech. 794, R3 (2016).

[31] S. Remmler and S. Hickel, Direct and large eddy simulation of stratified turbulence, Int. J. Heat Fluid Flow 35, 13 (2012).

[32] S. Khani and M. L. Waite, Buoyancy scale effects in large-eddy simulations of stratified turbulence, J. Fluid Mech. 754, 75 (2014).

[33] S. A. Thorpe, An Introduction to Ocean Turbulence (Cambridge University Press, Cambridge, UK, 2007).

[34] P. Billant and J.-M. Chomaz, Experimental evidence for a new instability of a vertical columnar vortex pair in a strongly stratified fluid, J. Fluid Mech. 418, 167 (2000).

[35] P. Augier (private communication). 